\title{
Impaired speed encoding is associated with reduced grid cell periodicity in a mouse model of tauopathy
}

Thomas Ridler [1], Jonathan Witton [2], Keith G. Phillips [3], Andrew D. Randall [1], Jonathan T. Brown* [1]

1. Institute of Biomedical and Clinical Sciences,

University of Exeter Medical School,

University of Exeter,

Hatherly Laboratories,

Prince of Wales Road,

Exeter, EX4 4PS, UK

2. School of Physiology, Pharmacology and Neuroscience, University of Bristol,

University Walk,

Bristol, BS8 1TD, UK

3. Lilly UK

Erl Wood Manor

Windlesham,

Surrey, GU20 6PH, UK

*Corresponding author: Dr Jonathan Brown, Institute of Biomedical and Clinical Sciences, University of Exeter Medical School, College of Medicine and Health, Hatherly Laboratories, Prince of Wales Road, Exeter, EX4 4PS, UK, Tel: +441392725504, Email: J.T.Brown@exeter.ac.uk. 


\section{Abstract}

1 Dementia is associated with severe spatial memory deficits which arise from dysfunction in

2 hippocampal and parahippocampal circuits. For spatially-sensitive neurons, such as grid cells,

3 to faithfully represent the environment these circuits require precise encoding of direction and

4 velocity information. Here we have probed the firing rate coding properties of neurons in

5 medial entorhinal cortex (MEC) in a mouse model of tauopathy. We find that grid cell firing

6 patterns are largely absent in $\mathrm{rg} 4510$ mice, while head direction tuning remains largely intact.

7 Conversely, neural representation of running speed information was significantly disturbed,

8 with smaller proportions of MEC cells having firing rates correlated with locomotion in $\mathrm{rTg} 4510$

9 mice. Additionally, the power of local field potential oscillations in the theta and gamma

10 frequency bands, which in wildtype mice are tightly linked to running speed, was invariant in

11 rTg4510 mice. These deficits in locomotor speed encoding likely severely impact path

12 integration systems in dementia. 


\section{Introduction}

Accurate spatial navigation requires the integration of sensory information to generate neural representations of space. Various high-level representations of the external environment are expressed at a single cell level within the extended hippocampal formation (hippocampus proper, dentate gyrus, entorhinal cortex and subiculum) and connected brain areas, regions well known to be critical for spatial memory. For example, place cells in the hippocampus fire action potentials in specific spatial locations ${ }^{1}$, whilst grid cells in the medial entorhinal cortex (MEC) fire in a highly organised, hexagonally distributed spatial pattern across an environment ${ }^{2,3}$. These directly spatially-sensitive neurons are collocated within the MEC with other functionally defined cell types, including head-direction ${ }^{4-7}$, speed $^{8,9}$ and border cells ${ }^{10,11}$. Together these are thought to provide the crucial computational information required for effective path integration, the process of using idiothetic cues to continuously calculate update positional information ${ }^{12}$.

Patients with Alzheimer's disease $(A D)$ and other dementia-spectrum disorders exhibit profound disruption in spatial navigation and memory, even at very early stages of the diseas $^{13-17}$. At a pathological level, misfolded tau deposition typically occurs first in the entorhinal cortex and hippocampus ${ }^{18}$. Taken together, these clinical signs strongly implicate pathology-induced circuit-level dysfunction in the hippocampal formation as a key early-stage functional deficit in AD. In this regard, there is substantial evidence from transgenic mouse models that dementia pathologies, such as $\beta$-amyloid deposition (A $\beta$ ) and hyperphosphorylation and misfolding of tau can disrupt the intrinsic properties ${ }^{19-22}$ and synaptic microcircuits ${ }^{23-25}$ of pyramidal cells in CA1 of hippocampus. Furthermore, there is growing evidence that place cells in the CA1 region of APP and tau overexpressing mice have reduced spatial-sensitivity ${ }^{19,26,27}$, strongly suggesting a failure of some aspects of the upstream functional circuits involved in spatial cognition. Recent evidence suggests that the circuits required for generation of theta and gamma frequency oscillations in the dorsal entorhinal cortex are especially prone to dysfunction in a mouse model of tauopathy (rTg4510 mice) $)^{28}$. 
41 Furthermore, Fu et al., (2017) showed that grid cell spatial periodicity is reduced in a mouse

42 in which tau overexpression is restricted to the entorhinal cortex. These data correlate with human imaging studies which suggest deficits in grid cell-like activity in the entorhinal cortices of people at genetic risk of developing $A D^{30}$.

Importantly, the effect of tau pathology on other functional components of the spatial navigation system less well understood. In this study we report for the first time that disruption to speed encoding in the MEC of rTg4510 mice may underlie deficits in grid cell function. Using high-density silicone probes and tetrode recording approaches in freely moving animals, we report that in both the medial entorhinal cortex and the CA1 region of the hippocampus, encoding of speed information at both the local field potential and cellular level is substantially impaired. We propose a model whereby such deficiencies in speed encoding networks result in loss of grid cell firing patterns in the MEC.

\section{Results}

\section{Tau pathology is associated with loss of oscillatory speed coding}

Neural coding of spatial information is likely to require the precise representation of locomotor speed $^{12,31-33}$. Velocity information can be represented in the brain via the dynamic regulation of the power and frequency of neuronal network oscillations in the theta and gamma frequency bands $^{34-38}$. We hypothesised that the profound deficits in spatial memory that occur in response to tau pathologies ${ }^{19,29,39}$ arise from impaired representation of locomotor speed in the hippocampal formation, ultimately leading to deficits in the encoding of spatial information. To examine this hypothesis we implanted multi-site silicone-based recording probes in the dorsal MEC of male wildtype (WT) and rTg4510 mice (6-7 months). Following a post-surgical recovery period, mice were encouraged to explore a familiar linear track, baited by food rewards at either end to encourage running between the two ends, whilst connected to a multichannel electrophysiological recording apparatus via a lightweight tether cable. 
As expected, in WT mice, theta oscillation properties followed changes to locomotor activity (fig 1A/B). Pooled data illustrate a clear relationship between running speed and theta oscillation power (linear regression: $R^{2}=0.71, p<0.001, n=7$ mice) and frequency (linear regression: $R^{2}=0.69, p<0.001, n=7$ mice) (fig $1 \mathrm{Ci}$ ). In contrast, in $r T g 4510$ mice, theta oscillation amplitude was poorly correlated with locomotor activity and remained at consistent levels throughout recording sessions (linear regression: $R^{2}=0.15, p=0.02, n=8$ mice, fig 1Ci). Importantly the overall Z-transformed correlation coefficient and slope relationships for theta power-running speed relationships were significantly lower in $\mathrm{rTg} 4510$ mice when compared to WT controls (2-way repeated measures ANOVA, see Table 1 and Figure 1D). Although less pronounced than in WT animals, peak theta frequency was correlated with running speed in rTg4510 mice (linear regression: $R^{2}=0.74, p<0.01, n=8$ mice, Figure 1Cii) which, across the population was not significantly different from WT (Correlation (Z'); WT: 0.84 $\pm 0.24, r \operatorname{Tg} 4510: 0.40 \pm 0.14$, unpaired T-test, $\mathrm{p}=0.13, \mathrm{n}=7 / 8$ mice), with no difference in the slope of the frequency-running speed relationship (Correlation $\left(\mathrm{Hz} / \mathrm{cms}^{-1}\right)$; WT: $0.045 \pm$ 0.0077, rTg4510: $0.037 \pm 0.011$, unpaired T-test, $\mathrm{p}=0.53, \mathrm{n}=7 / 8$ mice) .

Similar running speed modulation has been observed in both in the fast $(60-120 \mathrm{~Hz})$ and slow (30-50 Hz) gamma frequency bands ${ }^{36,37}$. In WT mice, both fast and slow gamma oscillation power was positively correlated with running speed (linear regression; fast gamma: $R^{2}=0.90$, $p<0.001, n=7$ mice; slow gamma: $R^{2}=0.17 p=0.013, n=8$ mice), although the slope of this association was greater for fast gamma frequencies (slow gamma: $27.1 \pm 8.4 \mathrm{mV}^{2} / \mathrm{Hz} . \mathrm{cm}^{-}$ 1, fast gamma: $80.6 \pm 19.2 \mathrm{mV}^{2} / \mathrm{Hz} \cdot \mathrm{cm}^{-1}$; 2-way repeated measures ANOVA; Table 1 and Figure 1D). rTg4510 mice did not show significant correlations for fast gamma frequency band oscillations (linear regression; fast gamma: $R^{2}=0.03, p=0.67, n=8$ mice, Figure 1D), but did for slow gamma frequencies $\left(R^{2}=0.43, p<0.01, n=8\right.$ mice, Figure 1D). However, compared to the WT population, this correlation was significantly lower only in the higher gamma frequency range (2-way repeated measures ANOVA, Table 1 and Figure $1 \mathrm{D}, \mathrm{n}=7 / 8$ mice). 
rTg4510 mice display hyperactive phenotype

rTg4510 mice have been shown to display a hyperactive phenotype under various conditions, which becomes more pronounced with developing tau pathology ${ }^{40-42}$. Since the experiments shown here display data that is heavily influenced by running speed, it was therefore important to observe this effect in the current experimental subjects. Under these recording conditions, rTg4510 mice also displayed a hyperactive phenotype. rTg4510 mice were shown to spend more time at faster running speed, with average speeds across recording sessions greater than WT control mice ( $P=0.04$, Unpaired T-test, $n=8 / 10$, Figure $S 1)$.

\section{Loss of grid rhythmicity in rTg4510 mice}

Impairments to the theta oscillatory code for running speed may impair grid cell rhythmicity. To establish whether this was the case, we used high density silicone probes to isolate a total of 279 single-units in layer II/II of the dorsal MEC from 10 mice (WT:150 units from 5 mice, rTg4510: 129 units from 5 mice). In this study we focused on the dorsal MEC, a subregion which has previously been identified as being particularly vulnerable in this transgenic model ${ }^{28}$. By performing post-hoc electrolytic lesions on each recording shank to identify probe location, we were able to estimate individual unit location by determining the largest average waveform along the $200 \mu \mathrm{m}$ probe for each isolated unit. Importantly, estimated recording location was not different between genotypes (Median distance from dorsal entorhinal border (interquartile range (IQR)): WT: 350(350) $\mu \mathrm{m}, \mathrm{rTg} 4510: 385(275) \mu \mathrm{m}, \mathrm{U}=9660, \mathrm{p}=0.77$, Mann-Whitney $\mathrm{U}$ test; Figure S2A).

Considered across the entire cellular population, MEC single units recorded from rTg4510 mice exhibited a small decrease in mean firing rate (Median (IQR): WT: 2.97 (16.7) Hz, rTg4510: $1.03(3.0) \mathrm{Hz}, \mathrm{U}=4472, \mathrm{p}=$ 0.01, Mann-Whitney $\mathrm{U}, \mathrm{n}=150 ; 129$ units, Figure S2E). As in the CA1 region of the hippocampus ${ }^{19}, \mathrm{rTg}_{4} 410$ cells also showed a pronounced decrease in theta modulation of firing patterns (Median (IQR) theta modulation index: WT: 9.17 (13.9), rTg4510: 2.29 (2.81), $U=2296, p<0.001$, Mann-Whitney $U, n=150 ; 129$ units, 
Figure S2C). Individual units with a theta modulation index (TMI) greater than $5^{43}$ were assigned as 'theta modulated'. While the majority of WT MEC cells $(65 \%)$ showed significant theta modulation, only a small proportion (13\%) passed threshold in rTg4510 mice $\left(X^{2}(1)=\right.$ 64.1, $p<0.0001$, Chi-Square test, Figure S2D). Extracellularly-recorded spike waveforms from interneurons in the mEC have a significantly narrower spike widths when compared to excitatory neurons ${ }^{44}$. The probability distributions of single units spike widths recorded from the dorsal MEC in WT mice had a bimodal distribution (Figure S2F). Based on this distribution and previously published work $^{44}$, we classed units with spike widths $<0.35 \mathrm{~ms}$ as putative interneurons and those $>0.35 \mathrm{~ms}$ as putative excitatory cells. Using this approach we found that $47 / 150$ units $(31 \%)$ were classed as putative interneurons in WT mice, where as in rTg4510 mice a significantly smaller proportion of units were classed as putative interneurons $\left(19 / 129\right.$ units, $15 \% ; X^{2}(1)=16.0, p<0.001$, Chi-Square test; Figure S2G). However, it should be noted that in the presence of tau pathology these measures should be met with caution, since the effect of action potential dynamics under these conditions is not necessarily clear.

Consistent with the literature we found that $\sim 1 / 4$ of cells in the WT MEC had grid-like spatial firing patterns in a $0.8 \mathrm{~m} \times 0.8 \mathrm{~m}$ square arena. We calculated a grid score for each cell, based on the rotational symmetry of the $2 \mathrm{D}$-autocorrelations and found that $36 / 150$ cells $(24 \%)$ had grid scores higher than the $95^{\text {th }}$ percentile of the distribution produced from shuffled spike timestamps (threshold $=0.21$; Figure 2B). In contrast, in rTg4510 mice there was an almost complete breakdown of grid cell periodicity (3/129 units, $2.3 \%$, threshold: 0.26$)$, with animals displaying irregular, non-uniform, firing fields across recording environments $\left(X^{2}(1)=27.1, p\right.$ $<0.0001$, Chi-Square test, Figure 2A/C). Furthermore, the distribution of grid scores of all cells recorded from WT and rTg4510 mice was significantly different (Median (IQR) grid score: WT, 0.03 (0.3); rTg4510, 0 (0.12), $n=150 / 129$ units; $P<0.001$; Mann-Whitney U). that MEC neurons did not differ significantly between genotypes, either as a distribution (WT, 
-0.05 (0.71), $n=150 ; r T g 4510,0$ (0.24), $n=129$ units; $P>0.05$; Mann-Whitney $U)$ or in the proportion of cells that exceed threshold (WT: 10/150, rTg4510 6/129, $X^{2}(1)=0.52, p=0.47$ Chi-Square test; data not shown).

Tau pathology is associated with impaired speed coding in MEC single units

Deficits in running speed-oscillation relationships at a local field potential level suggest alterations in the neural representation of running speed in the MEC of rTg4510 mice. Recent evidence suggests the existence of a separate population of cells in the MEC which express a rate code for running speed ('speed cell') ${ }^{8}$. We calculated a speed score for all recorded cells, whilst mice ran on an L-shaped track, by computing the Fisher-transformed correlation coefficient $(z)$ between instantaneous firing frequency and running speed (Figure 3A-B). Units were considered 'speed-modulated' if they had a speed score outside the 5-95 centile range of a shuffled distribution of data produced from 250 shuffles for each cell (Figure 3C). In WT mice, $85 / 150$ ( $57 \%$ ) cells had firing rates significantly modulated by running speed, whereas in rTg4510 mice a significantly lower proportion of cells was speed modulated (17/129; $13 \%$; $X^{2}(1)=56.55, p<0.0001$, Chi-Square test, Figure 3E). As an overall population, $r \operatorname{Tg} 4510$ MEC units also displayed a significantly lower average speed score compared to WT mice (Median (IQR): WT: $z=0.11(0.31), r T g 4510: z=0.009(0.01), U=3603, p<0.0001$, MannWhitney $U, n=150 ; 129$ units, Figure 3D), with cells recorded from $r T g 4510$ mice, on average displaying a running speed correlation close to zero.

Speed modulated MEC neurons can be broadly split into those that display linear and saturating exponential relationships between running speed and firing rate ${ }^{9}$. To account for this, speed scores were calculated for log transformed data and further classified as linear or exponential by the best regression fit (Figure S3A). In WT animals speed-modulated cells showed an approximately equal distribution between linear and saturating running speed relationships (Figure S3B linear: 41/85 units, saturating: 44/85 units, which did not differ 
significantly in the remaining rTg4510 MEC neurons (linear: 10/17 units, saturating: 7/17 units, $X^{2}(1)=0.6, p=0.43$ Chi-Square test, Figure S3B).

Previous studies have suggested that a small proportion of speed-modulated cells decreased their firing frequency during locomotor activity ${ }^{8,9}$. On the linear track, in WT mice, this population was observed to be consistent with previous reports $(\sim 13 \%$ of speed modulated cells) $)^{8,9}$. In contrast, in rTg4510 mice the proportion of cells with negative speed relationships was substantially and significantly higher (WT: 11/85 units, rTg4510: $7 / 17$ units, $X^{2}(1)=7.8$, $p=0.005$, Chi-Square test, Figure S3C), meaning that speed modulated cells were split much more evenly between positive and negative associations with firing rate. Given that many MEC speed cells are fast spiking in nature ${ }^{8}$, we also assessed the spiking properties the recorded of speed-sensitive units. We found that in WT and $\mathrm{rTg} 4510$ mice, a similar proportion (41\% and $31 \%$, respectively; $x^{2}(1)=0.6, p=0.4$, Chi-Square test) of speed-modulated neurons were fast-spiking (mean firing rate $>10 \mathrm{~Hz}$ ) (Figure S3E). The proportion of all cells that were classified as fast-spiking was substantially lower in $\mathrm{rTg} 4510$ mice (13\% vs $30 \%$ in WT; $\mathrm{X}^{2}$ (1) $=19.0, \mathrm{p}<0.001$, Chi-Square test; Figure S3E), although this likely reflects the overall reduction in mean firing rate in these neurons (Figure S2E).

Speed modulated firing of single units has also been observed in the hippocampus ${ }^{8,46,47}$, so we next sought to determine whether deficits in speed tuning in rTg4510 mice were specific to the MEC, or were also represented downstream in the hippocampus proper. For this purpose, data were taken from previous single unit and local field potential recordings in the hippocampal CA1 region of $\mathrm{rTg} 4510$ mice (Booth et al., 2016b) at a similar age point and reanalysed to assess the contribution of locomotor activity to firing rate. In this region, CA1 theta band activity in the local field potential was also correlated with running speed in WT mice (linear regression; theta power; WT: $R^{2}=0.83, p<0.001, n=6$ mice), but not in $r T g 4510$ mice (linear regression: $R^{2}=-0.04, p=0.6, n=4$ mice, Figure S3A). A significant proportion of CA1 neurons had firing rates modulated by running speed in both WT and $\mathrm{rTg} 4510$ mice 
(WT: 25/46 units, rTg4510: 27/52 units). Importantly however, as seen in MEC recordings, a much greater proportion of CA1 cells were negatively modulated by locomotor activity in rTg4510 mice than in WT controls (WT: 5/25 units, rTg4510:13/27 units; $X^{2}(1)=4.5, p=0.03$, Chi-Square test, Figure S4F).

\section{Normal head-direction tuning in rTg4510 mice}

Another critical component of the path integration system in the MEC are cells which accurately encode directional heading information. Therefore, we next sought to establish whether head direction cells $s^{4,5}$ in the MEC were similarly disrupted in $\mathrm{rTg}^{4} 510$ mice. We calculated a head direction score (HD) by determining the mean vector length of circular firing distributions and, as with other functional metrics, we compared observed HD scores with shuffled distributions (Figure 4A\&B). Importantly, a similar proportion of MEC cells in WT and rTg4510 mice surpassed the $95^{\text {th }}$-centile threshold (WT: 19/150, rTg4510: 14/129, $X^{2}(1)=$ $0.79, p=0.37$, Chi-Square test, Figure $4 \mathrm{C})$. Furthermore, as a population, the HD score was actually slightly, but significantly, higher in rTg4510 cells compared to WT (Median (IQR) vector length: WT: 0.074(0.091), rTg4510: 0.11(0.12), $U=6509, p=0.0014$, Mann-Whitney $U, n=150 ; 129$ units, Figure 4B-E). These data suggest that head-direction tuning remains intact in $\mathrm{rTg} 4510$ mice. Similarly, there was no change in the proportion of border cells observed across genotypes (WT: 4/150, rTg4510: 6/129, $X^{2}(1)=0.79, p=0.37$, Chi-Square test; data not shown), with relatively small numbers of cells passing threshold produced from shuffled datasets. Neither was there a difference in the average border score between the two populations (Median (IQR) vector length: WT: 0.097(0.67), rTg4510: -0.04(0.78), U = 6191, p $=0.94$, Mann-Whitney $U, \mathrm{n}=150 ; 129$ units; data not shown).

\section{Decreased firing rate does not account for changes in spatial metrics}

Since overall firing rates of MEC neurons in rTg4510 mice are signifcantly slower than WT controls (Fig S2), we next asked whether these changes may account for differences in grid, speed, or head-direction tuning scores between genotypes. Spike trains for each WT cell were 
downsampled incrementily between 1 and 100 times. As reported previously ${ }^{48}$, healthy grid

237 cells are largely resistent to such decreases in firing, until firing rates are decreased to an average of $\sim 0.1 \mathrm{~Hz}$ (Figure S5A/B). This would represent a 10 fold greater change in firing (Median (IQR): WT: 2.97 (16.7) Hz, rTg4510: 1.03 (3.0) Hz, U = 4472, p = 0.01, Mann-Whitney $U, n=150 ; 129$ units, Figure S2E).

Impaired conjunctive representation of spatial information in rTg4510 mice

A proportion of cells within the MEC are known to demonstrate conjunctive functional representation ${ }^{5}$. Using the tuning-curve approaches defined above, we found that $\sim 60 \%$ of WT cells passed the threshold for only a single functional class of neuron (i.e. grid-, speed- or HD-cells). A further $25 \%$ of WT cells passed the threshold for more than one functional class and were therefore conjunctive cells, whilst the remaining $\sim 20 \%$ of cells were unclassified (Figure S6D). For example, in this study, only 3\% of spatially sensitive cells were pure grid cells, whilst the remaining grid cells also passed the threshold for speed- and HD-modulation (Figure S6C). In contrast, due to the substantial reduction in numbers of speed and grid cells, very few rTg4510 cells ( 2\%) expressed any conjunctive representation (Figure S6D). 


\begin{tabular}{|c|c|c|c|c|c|}
\hline \multicolumn{6}{|l|}{ Correlation (Z) } \\
\hline Source of Variation & Df & Sum-of-squares & Mean square & $\mathrm{F}$ & $\mathrm{P}$ \\
\hline Frequency & 2 & 0.470 & 0.235 & 4.341 & 0.051 \\
\hline Genotype & 1 & 0.602 & 0.602 & 5.927 & 0.038 \\
\hline Interaction & 2 & 1.330 & 0.665 & 8.379 & 0.005 \\
\hline \multicolumn{6}{|l|}{ Slope $\left(\mathrm{mV} 2 / \mathrm{Hz}_{\mathrm{cm}} \mathrm{cm}^{-1}\right)$} \\
\hline Source of Variation & Df & Sum-of-squares & Mean square & $\mathrm{F}$ & $\mathrm{P}$ \\
\hline Frequency & 2 & 0.004 & 0.002 & 8.973 & 0.004 \\
\hline Genotype & 1 & 0.008 & 0.008 & 86.224 & $>0.001$ \\
\hline Interaction & 2 & 0.005 & 0.003 & 16.623 & $>0.001$ \\
\hline
\end{tabular}

Table 1: Results of 2-Way repeated measure ANOVA for oscillation-running speed interaction. Results of relevant post-hoc pairwise-comparisons displayed on Figure 1. 
$\mathrm{Ai}$

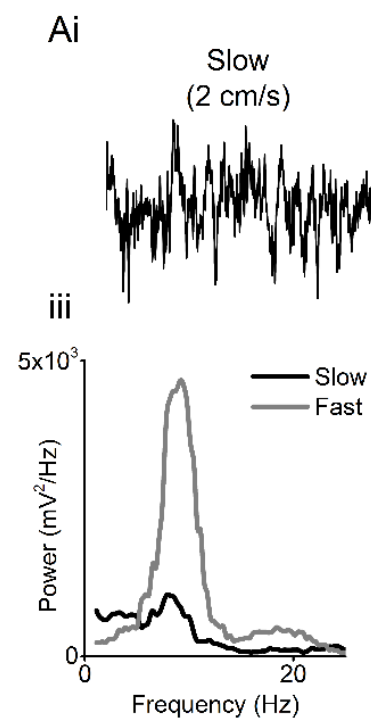

ii

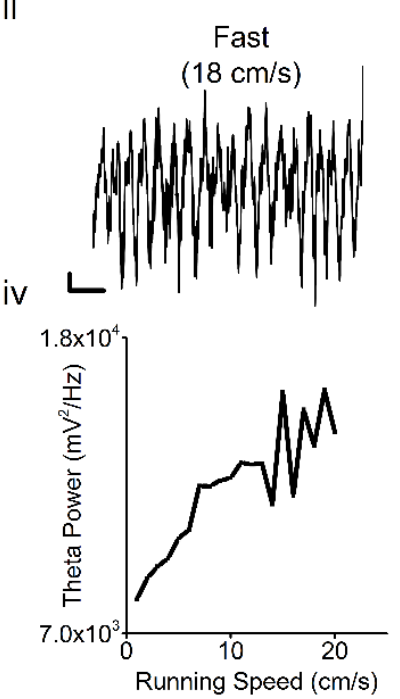

$\mathrm{Bi}$

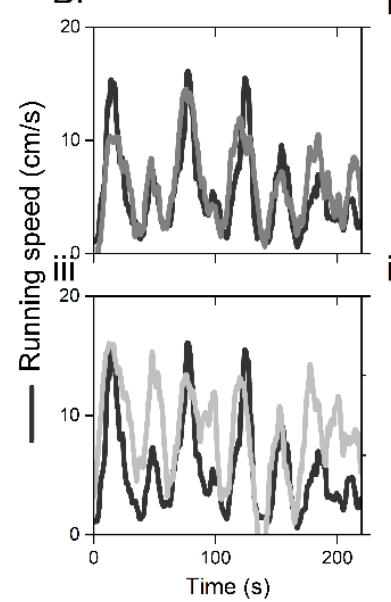

ii

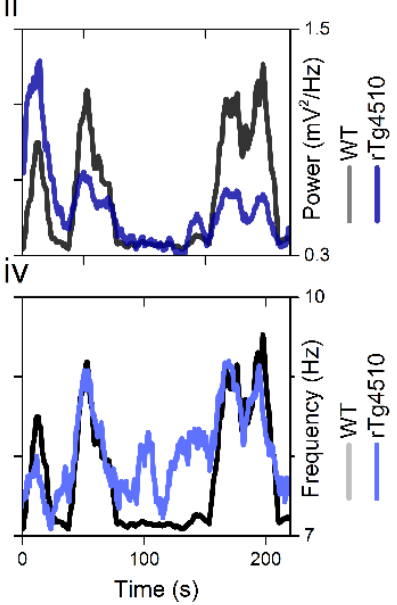

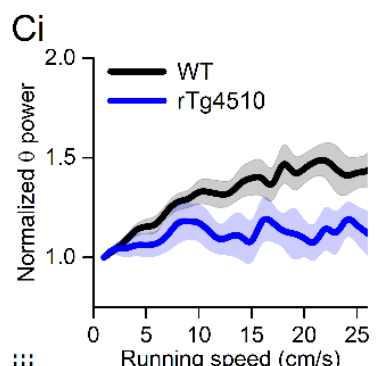

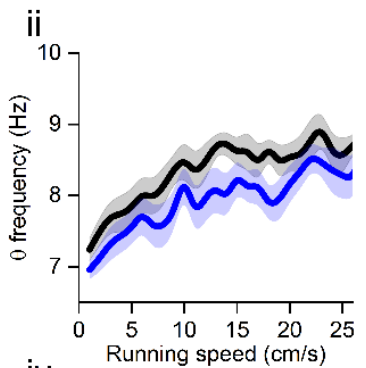

iii
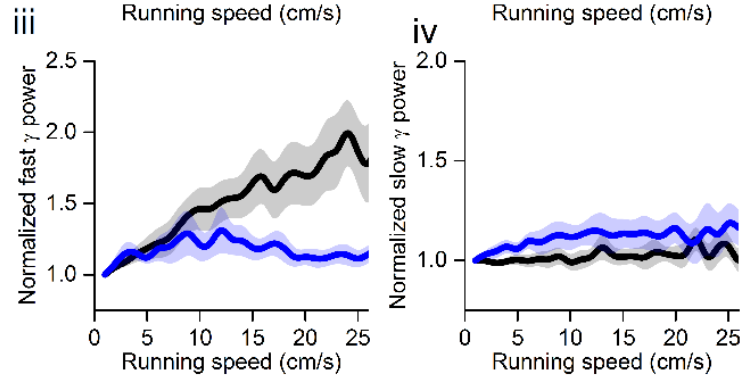

$\mathrm{Di}$
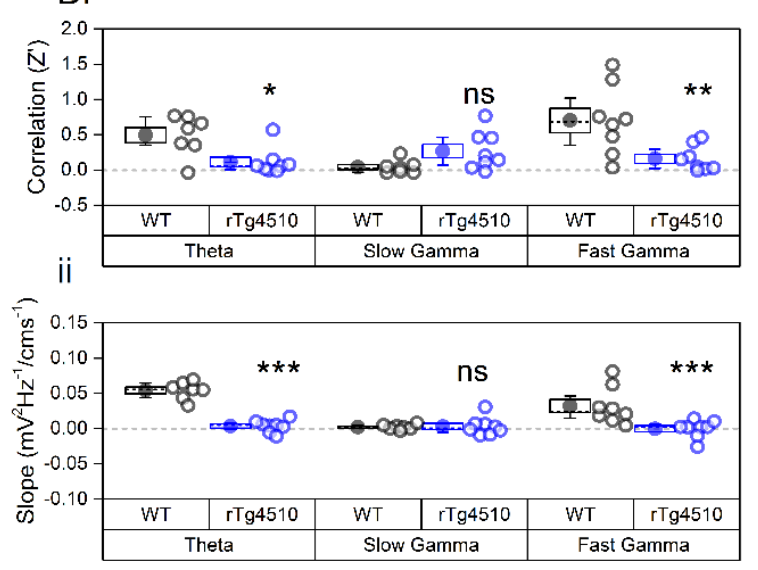

Figure 1: Oscillation-running speed relationship is impaired in rTg4510 mice. A) Local field potential from periods of slow (i) and fast (ii) running speed showing faster and larger theta oscillations during locomotor activity. iii) Power spectra of data shown in i and ii for slow (black) and fast (grey) running periods. iv) Example relationship between running speed and average theta oscillation power across recording session. B) Example plots showing animals running speed on linear track (black, left $Y$ axis) showing high correlation with theta oscillation (grey, right $\mathrm{Y}$ axis) power (i) and frequency (iii) over several minutes of recording. Corresponding example from rTg4510 mouse showing with theta oscillation amplitude (ii) and frequency (iv) with decreased association with running speed. C) Running speed -theta oscillation relationships for power (i; normalised to $1-2 \mathrm{~cm} / \mathrm{s}$ bin) and frequency (ii). Also shown are fast (iii) and slow (iv) gamma power-running speed relationships. D) Pooled data for each animal showing Z-transformed correlation coefficients (i) and slopes (ii) of running speed-oscillatory power relationships for different frequency bands $\left({ }^{*} p<0.05,{ }^{* *} p<0.01,{ }^{* * *} p\right.$ $<0.001$, ns = not significant, Bonferroni-corrected pairwise-multiple comparisons; for 2-way repeated measures ANOVA main effects and interactions see Table 1). 

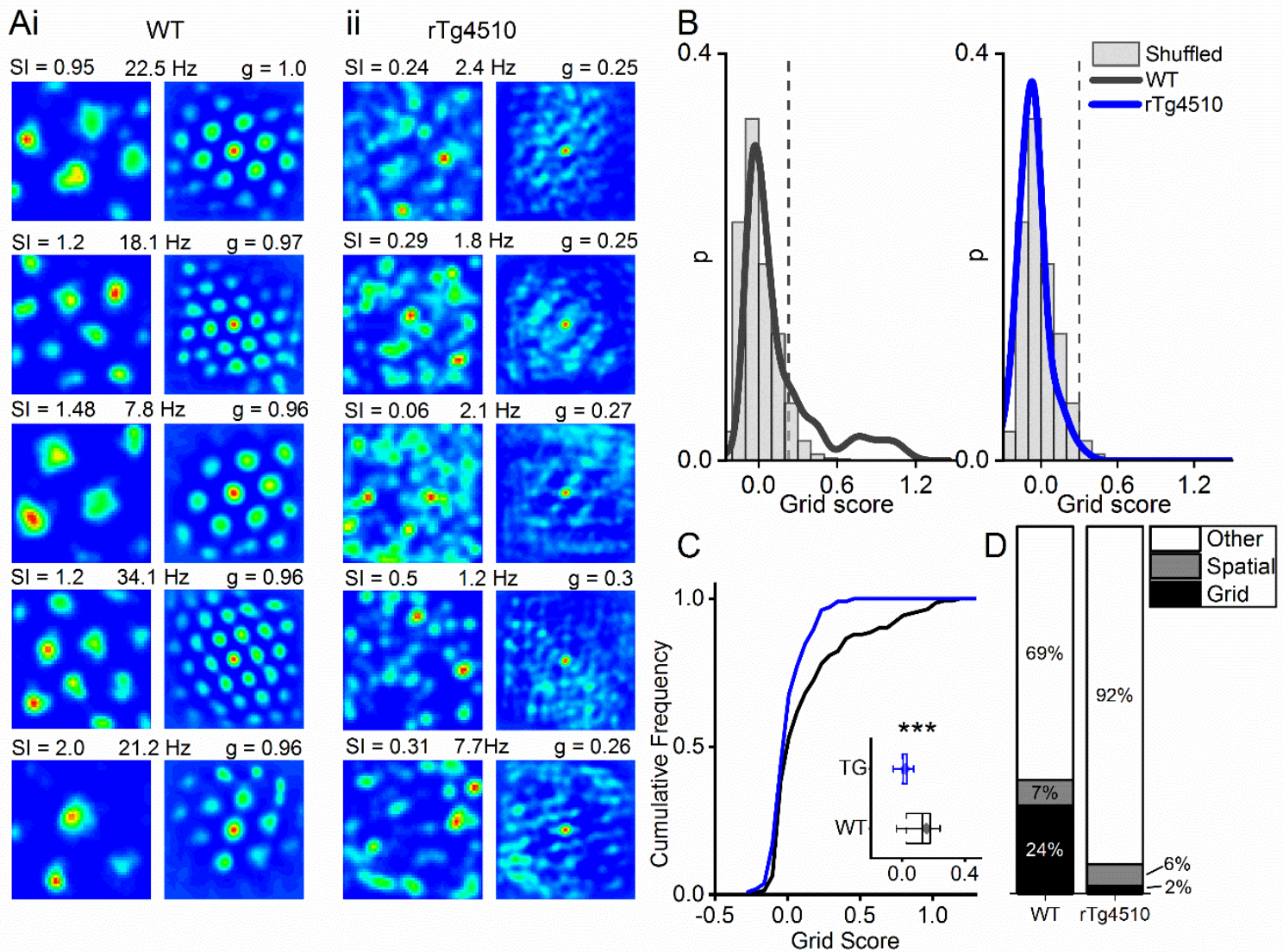

Figure 2: Breakdown of grid cell periodicity in rTg4510 mice. A) Example spatial firing patterns (left) and 2D autocorrelations (right) of cells from WT (i) and rTg4510 (ii) mice in a $0.8 \times 0.8 \mathrm{~m}$ square arena, displayed with grid score $(\mathrm{g})$, spatial information content (SI) and peak firing rate across recording environment. 5 cells with the highest grid score displayed for each genotype. B) Histograms of grid scores for all single units recorded from WT and rTg4510 mice. Observed data plotted as a solid line, shuffled data shown as grey bars. The $95^{\text {th }}$ centile of shuffled distributions is plotted as a dashed line. C) Cumulative frequency plots for grid score; inset box plot illustrating average values for each genotype (dotted line: median, diamond: mean \pm SEM, whiskers: $25^{\text {th }} / 75^{\text {th }}$ centile), ${ }^{* * *} p<0.001$, Mann-Whitney U test. D) Proportions of grid and spatial non-grid cells greater than threshold in WT and rTg4510 mice. 


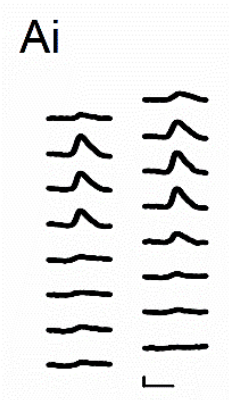

$\mathrm{Bi}$

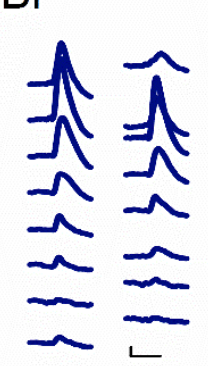

$\mathrm{Ci}$

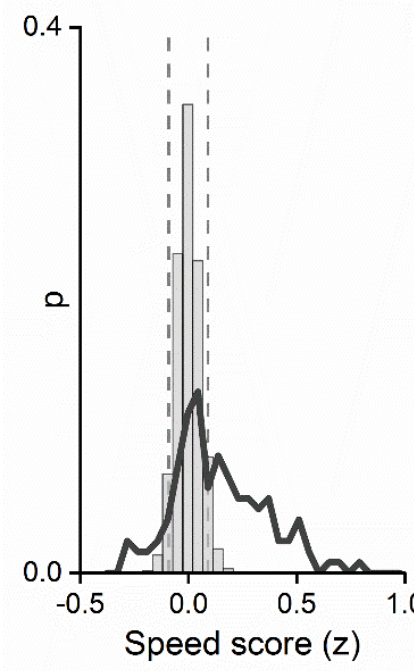

WT

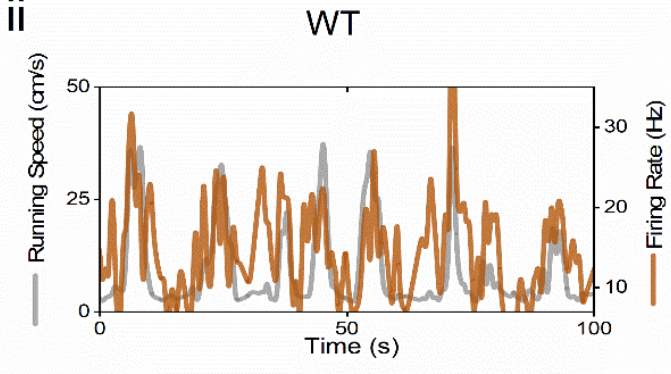

ii

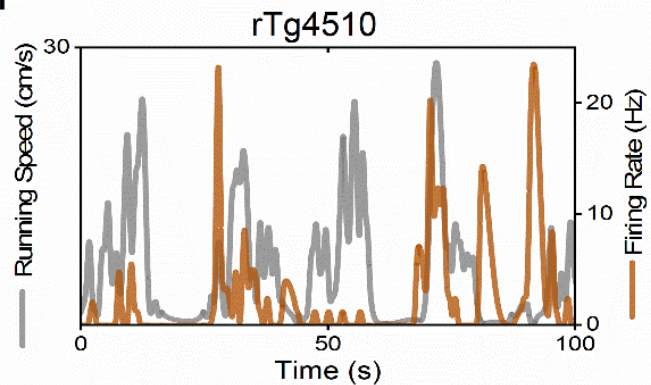

iii

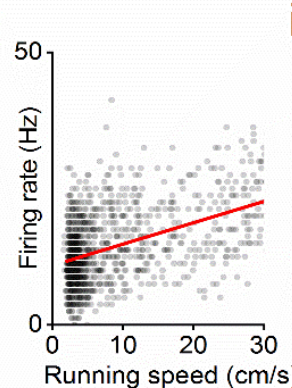

iii

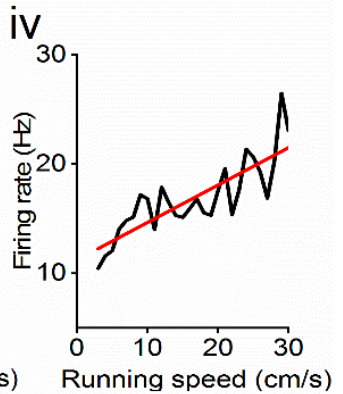

iv

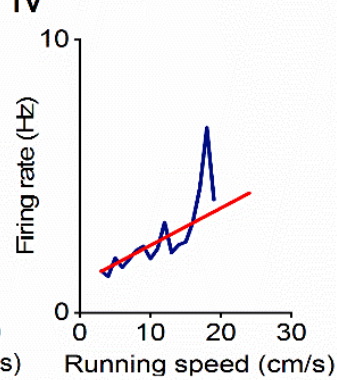

ii

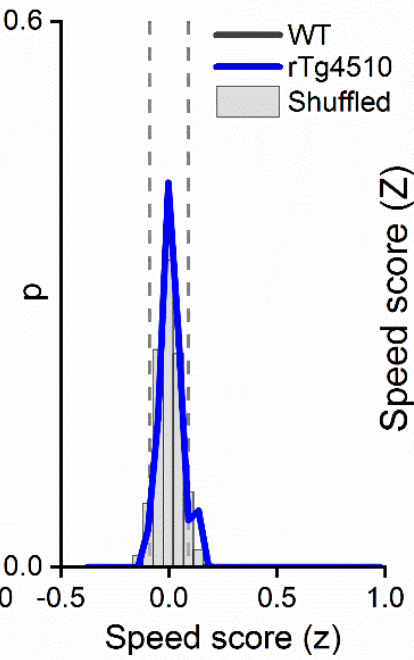

D

E
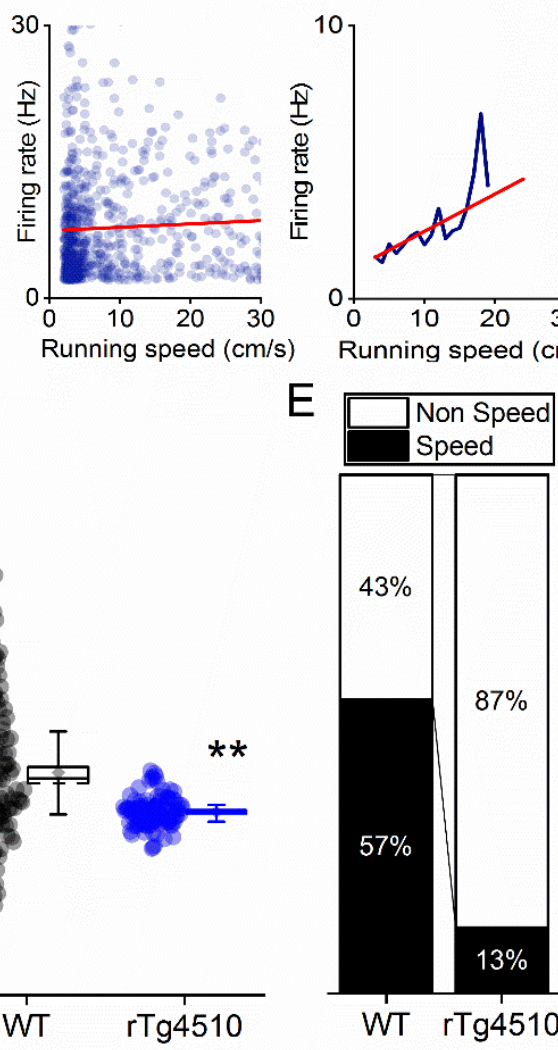

Figure 3: Decreased speed modulation of MEC single units in rTg4510 mice. Average waveforms of a single unit recorded from a 16 channel shank silicone probe, from a WT (Ai; black) and rTg4510 (Bi; blue) mouse. The running speed (grey) and cell firing rate (orange) (ii), correlation between running speed and firing rate for each time bin (40 ms) (iii), and average for each speed bin $(1 \mathrm{~cm} / \mathrm{s})$ (iv) are shown for each of these example cells. Red line: linear fit for each. Scale bars: $0.3 \mathrm{~ms}, 25 \mu \mathrm{V}$. C) Distribution of speed scores for WT (i) and rTg4510 units (ii) with shuffled distribution of scores (grey bars); $5^{\text {th }} / 95^{\text {th }}$ centile threshold: dotted lines. D) Average speed score for each recorded MEC unit. Box plots: dotted line: median, diamond: mean \pm SEM, whiskers: $25^{\text {th }} / 75^{\text {th }}$ centile), ${ }^{* *} p<0.01$, Mann-Whitney U test. E) Proportion of cells classified as speed modulated $\left(>95^{\text {th }}\right.$ or $<5^{\text {th }}$ centile of shuffled distribution). 


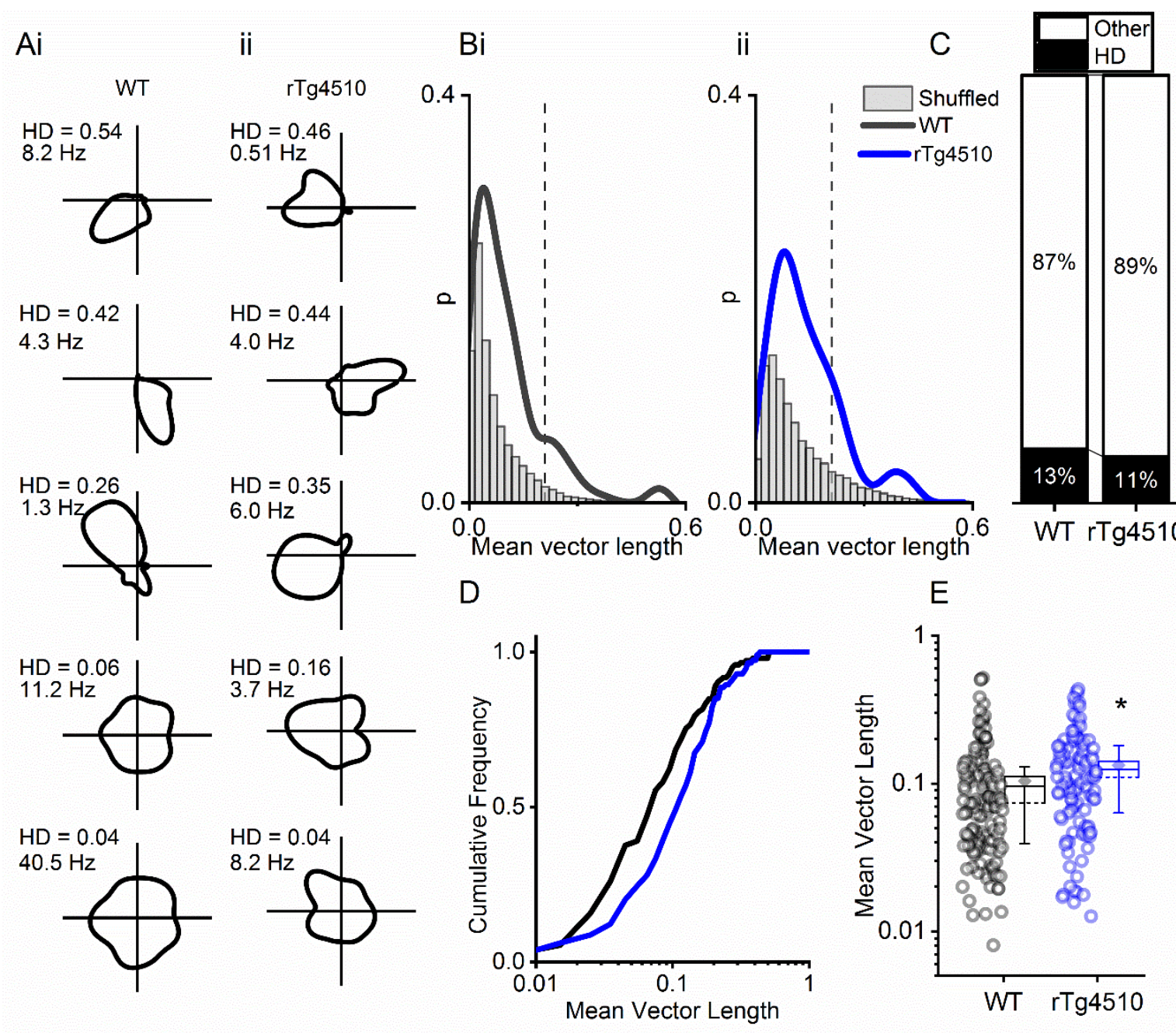

Figure 4: rTg4510 mice retain MEC head-direction tuning. A) Example head-direction tuning of 5 WT and 5 rTg450 units, displayed with head direction score (HD) and peak firing rate across head direction bins. B) Distribution of HD scores for WT (i) and rTg4510 mice (ii) with shuffled distribution of scores (grey), $95^{\text {th }}$-centile threshold: dotted line. C) Proportions of cells with head direction scores over threshold in WT and rTg4510 mice. D) Cumulative frequency distribution of HD scores (mean vector length). E) Mean vector length for all cells, showing a small but significant increase in head-direction tuning across the population in rTg410 mice (dotted line: median, diamond: mean \pm SEM, whiskers: $25^{\text {th }} / 75^{\text {th }}$ centile), * $p<0.05$ Mann-Whitney U test. 


\section{Discussion}

262

263

264

265

266

267

Continuous integration of running speed information in the MEC has been proposed to be critically important for spatial navigation and path integration $n^{5,8,31,49}$. The current experiments clearly show that unlike WT animals, rTg4510 mice do not express adequate representations of locomotor activity in the MEC local field potential (LFP), since theta and gamma oscillations display blunted, or absent, relationships with running speed.

There has been some suggestion in the literature that dementia pathology affects the firing pattern of grid cells in the MEC both in mice ${ }^{29}$ and grid-like neural representations in humans ${ }^{30}$. To date, however, no studies have examined changes to speed modulated firing of MEC single units in tauopathy. Fu et al., (2017) also describe no effective impact of tau pathology on headdirection or border cell firing rates using the rTgTauEC mouse model. However, it should be noted that in this case tau pathology was restricted to the MEC, rather than impacting the broader circuits involved in these processes. Our data suggest the hypothesis that reduced grid cell periodicity may be the result of the impaired integration of running speed information in the MEC. While the causality of this is not clear, it is evident that both are perturbed in this tau model, which is likely to have profound influences on spatial information processing.

\section{Vulnerability of grid cell firing}

These results highlight the importance of an intact path integration system in maintaining grid cell periodicity. Several studies have shown a breakdown of grid cell firing patterns after the inactivation of important spatial information streams. For example, inhibition of the medial septum, which controls theta rhythmicity, but also speed modulated inputs, produces a complete breakdown of grid cell periodicity ${ }^{50,51}$. Likewise, inactivation of the anterior thalamic nuclei (ATN) disrupts head direction tuning in the MEC, also impairing the grid cell signal ${ }^{52}$. 
Inactivation of reciprocal hippocampal inputs into the MEC is also sufficient to produce a breakdown in grid cell periodicity ${ }^{48}$.

The almost complete breakdown of grid cell firing in rTg4510 mice contrasts with the effect on spatial representation in the hippocampus of these animals. While several studies have shown a reduction in the spatial information and stability of hippocampal place cells ${ }^{19,26}$, firing fields are still consistently present in these mice. Given the pattern of degeneration across the hippocampal formation ${ }^{39,53-55}$, poor place representation ${ }^{19,26}$ may be the result of weakened entorhinal inputs ${ }^{56-58}$. Since hippocampal place cells could be thought of as conjunctive integrators of multiple spatial (and non-spatial) input streams, surviving inputs may facilitate encoding of an impoverished spatial representation in these mice ${ }^{19,26}$. However, the loss of grid/speed input prevents this from being anchored to an egocentric spatial reference frame, resulting decreased spatial stability. Presumably, whatever representation is being encoded by place cells is anchored to an allocentric reference frame. Decreasing the availability of visual/contextual cues or implementing a pure path integration task would likely exacerbate spatial learning/memory impairments. Reductions in grid and place field activity may therefore mirror their appearance in neuronal development, where place cells appear before grid cells but mature fully only after grid cell development ${ }^{59,60}$. The precise temporal nature of grid and place cell impairment in this model is as yet unknown. However, taken together, these data further suggest that hippocampal neurons can form place fields in the absence of effective grid cell firing.

Grid cells are proposed to play a key role in path integration ${ }^{31,32,61,62}$. For example, mice lacking GluA1-containing AMPA receptors have been shown to display reduced grid cell periodicity, correlating with impairments on a path integration-based task ${ }^{63}$. Path integration has also been suggested to be impaired in populations of dementia and $\mathrm{MCl}$ patients ${ }^{13,15,17}$. The direct 
association between these two factors is still unclear; however, it is likely that grid cell deficits, as described above, directly contribute to the deficits in memory in $\mathrm{rg} 4510$ mice $^{19,53}$.

\section{Firing rate changes in rTg4510 mice}

These data show large differences in firing rates between WT and rTg4510 mice, which may have the capacity to bias measurements such as the grid score. However, while most manipulations that disrupt grid firing also decrease entorhinal firing rates, it is important to note that 'healthy' grid scores are largely resistant to moderate to large decreases in firing rate. By down sampling the spike trains of MEC neurons we show that decreased firing alone is not sufficient to account for changes in spatial metrics seen here. It is more likely, for example, that changes to theta rhythmicity, which often accompany the breakdown of grid cell periodicity, are responsible for changes in these mice.

\section{Negative speed modulation of MEC neurons}

In WT animals, only a small population of MEC neurons display negative relationships with running speed ${ }^{8,9}$. These data show that during recordings on the linear track, rT4510 mice have a larger proportion of cells that decrease their firing rate with increases in running speed. Interestingly, neurons in the medial septum also display a more even split between positive and negative speed modulation ${ }^{64}$, suggesting that, under normal physiological conditions, the MEC does not simply recapitulate these firing patterns, but integrates this information differently. The precise targets of negatively speed modulated inputs into MEC are unclear; however it is possible that they are more likely to be conjunctive with other spatial information systems, such as grid encoding or head direction. Indeed, negatively speed modulated cells are more likely to be tuned to head direction ${ }^{9}$, which is consistent with the observed increase in mean vector length observed in $\mathrm{rTg} 4510$ neurons at a population level (Figure 4). 
339 A further feature of the inactivation studies described above ${ }^{48,50-52}$ is the stability of head 340 direction tuning in the MEC. Blocking medial septum activity impairs grid cell firing, but not 341 head direction tuning ${ }^{50,51}$. Interestingly, the breakdown of grid periodicity after hippocampal 342 inactivation has also been shown to reveal head direction tuning in grid cells that would previously not have been modulated by head direction ${ }^{48}$. It is possible that the small but significant population increase in head direction tuning in $\mathrm{rTg} 4510$ mice may reflect this unmasking of head direction inputs from cells that would have previously displayed grid patterns. However, this is a hypothesis that would need to be addressed directly with long term recordings of grid cells as tau pathology increased to critical levels to impair spatial firing patterns. This approach is unlikely to be achieved with current technology. In any case, the arrival of head direction information into the MEC is likely the result of an anatomically distinct pathway ${ }^{65}$ that appears to be relatively preserved in the face of the tau pathology burden in rTg4510 mice at this stage. This is most likely due to a greater dependence on subcortical structures, such as the ATN, that integrate vestibular information ${ }^{66-68}$.

Theta oscillations show increases in both amplitude and frequency depending on running speed (Figure 1). Spatially modulated MEC neurons are required to integrate large quantities of multimodal sensory information from their environment. At fast running speeds, the time window for this integration is smaller and it may therefore be necessary to increase the sensitivity of such neurons during locomotion to accurately retrieve spatial associations from memory. In rTg4510 mice, theta oscillation power appears to be independent of running speed 
In WT mice, the gamma oscillation power relationship with running speed shows a strong predominance for fast gamma frequencies (Figure 1). This is consistent with the proposed information flow across the hippocampal formation, in which the MEC provides the input responsible for fast gamma frequencies in CA1 region of the hippocampus ${ }^{69}$. While the CA1 area has been shown to display two distinct peaks in the gamma band power spectra, MEC LFP predominantly contains faster gamma frequencies only ${ }^{69,70}$. It is perhaps not surprising then, that differences between genotypes (Figure 1) are only observed at these faster frequencies. For this reason also, conclusions regarding the slow gamma oscillation frequency should be made with caution. While slow gamma frequency has previously be shown to display a negative speed relationship in the hippocampus, MEC correlations are generally thought to be positive 36,71 .

It is also worth noting that $r \operatorname{Tg} 4510$ mice, like several other models of dementia, exhibit a hyperactive phenotype ${ }^{55,72-74}$, and hence altered patterns of locomotor activity. This may reflect dysregulation of the septohippocampal pathway, activation of which has been shown to stimulate movement or alternatively may reflect dysfunction in motor-related brain regions. Nevertheless, whilst it should be noted that rTg4510 mice are experiencing their environment at a generally higher state of arousal, at least in terms of locomotor activity, the key finding of this study is that this locomotor activity is improperly represented in the hippocampal formation. Conclusions

Overall, these data show a clear breakdown in grid cell periodicity in rTg4510 mice compared to WT controls. In addition, they suggest a role for the dysfunctional processing of locomotor activity in this process, since the representation of running speed information in MEC single units is severely disrupted in these mice, while head direction tuning remains constant, or slightly increased. The changes to MEC single unit firing are likely to have profound implications for the impairments in spatial memory observed in these mice and suggest observable parameters to assess in dementia patient populations, for example through speed modulated $\mathrm{fMRI}$ signals in virtual environments. 


\section{Methods}

Animals

392

All procedures were carried out in accordance with the UK Animal (Scientific Procedures) Act 1986 and were approved by the Universities of Exeter and Bristol Animal Welfare and Ethical Review Body.

397 All surgical procedures were conducted using standard sterile and aseptic techniques. Animals were anaesthetized using isoflurane (4\%) and fixed into a stereotaxic frame (ASI instruments). Anaesthesia was reduced and maintained at 1-2\% during surgery. After careful cleaning of the skull surface, small screws (Antrin Miniature Specialities) were inserted into each bone plate in order to anchor the electrode array. Silver wire (World Precision Instruments) was soldered to a screw overlying the cerebellum to be used as a ground.

Probes were implanted at $0.2-0.3 \mathrm{~mm}$ anterior to the transverse sinus and $3-3.25 \mathrm{~mm}$ from midline. Linear probes were implanted and fixed $3 \mathrm{~mm}$ below the dura mater and angled at 10 degrees in the posterior to anterior direction in the sagittal plane in order to record consistently from layer II/III along the dorsal-ventral axis of the MEC. High density 16- (Neuronexus) or dual shank 32-channel (Cambridge Neurotech) silicone probes were implanted 0.3-0.5 mm below dura at an angle of 5 degrees, also in the posterior direction and subsequently moved slowly into the cortex using their attached microdrive (Cambridge NeuroTech). RelyX Unicem skull and anchor screws. 
Animals were given at least 1 week of post-operative recovery before initial recording sessions. Local field potential (LFP) signals were recorded using a Digital Lynx 10S recording system (Neuralynx, Bozeman, MT, USA) tethered to a HS-18 or HS-36 unity gain headstage and Cheetah 5 data acquisition software (Neuralynx). The headstage and tether were counterbalanced using a moveable, weighted arm to allow for the maximum flexibility of movement. Two light-emitting diodes (LEDs) on the headstage and an overhead video camera (sample rate $25 \mathrm{~Hz}$ ) were used to continuously track the animals' location using Cheetah's built in video tracking software (VTS), allowing estimation of position and therefore running speed. Once recorded, invalid tracking points, i.e. time-points where no light threshold was reached, were excluded and the animal's position interpolated from the two nearest points. Estimation of running speed was performed on binned position data, with erroneous bins, above $50 \mathrm{~cm} / \mathrm{s}$, also removed. LFP data were recorded while animals explored either a linear track $(1.5 \mathrm{~m})$ or square open field $(0.8 \mathrm{~m} \times 0.8 \mathrm{~m})$.

Data were continuously sampled at $2 \mathrm{kHz}$, band-pass filtered $(1-500 \mathrm{~Hz})$ and stored on a PC for offline analysis. All LFP signals were analysed in MATLAB, using open-source toolboxes or custom routines utilising built-in functions. Multi-tapered spectral analysis was performed using the Chronux toolbox (available at http://chronux.org/).

Power and peak frequency of LFP frequency bands were compared to running speed. Spectral analysis was conducted on $0.5 \mathrm{~s}$ bins of LFP data and compared to running speed calculated from the same time frame. For running speed curves, locomotor activity was divided into 1 $\mathrm{cm} / \mathrm{s}$ bins (between $1-30 \mathrm{~cm} / \mathrm{s}$ ) and oscillatory power and peak frequency averaged across all 
relevant sections of data. Theta and gamma oscillation power was normalised to the power in these frequency bands during non-movement, defined as speeds under $1 \mathrm{~cm} / \mathrm{s}$.

\section{Analysis of single unit data}

For single unit data, recordings were referenced to the ground electrode, continuously sampled at $40 \mathrm{kHz}$, bandpass filtered between 1-30 kHz and saved unprocessed on a PC for offline analysis. Each channel was referenced offline to a common-average of the opposite 16-channel shank ( $250 \mu \mathrm{m}$ away) in order to eliminate signals common across the electrode array such as noise and movement artefacts. Extracellular spike activity was detected and sorted using the klusta open source software package found at: http://klusta.readthedocs.io/en/latest/ $^{75}$. Clusters were classified as either putative interneurons or putative excitatory cells (pyramidal or stellate cells) by their spike half-width, taken from the peak to the subsequent trough of the average extracellular waveform. While the majority of cells recorded in the MEC are excitatory, a significant population can be classified as inhibitory interneurons ${ }^{44,76,77}$. Using the average spike waveform, putative interneurons were classified as displaying a spike-width less than $0.35 \mathrm{~ms}$, based on the extracellular properties of PV+ interneurons isolated optogenetically ${ }^{44}$. This approach was taken alone, rather than in combination with average firing rare of neurons, since MEC interneurons have been shown to vary widely in their spike frequency ${ }^{44}$. Neurons were described by a theta modulation index (TMI), based on the fast Fourier transform (FFT) of spike-train autocorrelations, using methods described previously ${ }^{19,43,78}$. Autocorrelations were produced with $\pm 500 \mathrm{~ms}$ lags and $2 \mathrm{~ms}$ bin size. The peak at 0 lag was reduced to the next maximal value and the entire function mean-normalised by subtracting the mean from all values. The autocorrelation was tapered using a Hamming window to reduce spectral leakage and FFT calculated. The power spectrum was calculated by dividing the square of the FFT by the transform length $\left(2^{16}\right.$, scaled to the length of the autocorrelation). TMI was defined as the mean power within $1 \mathrm{~Hz}$ of each side of the peak in the theta frequency range $(5-12 \mathrm{~Hz})$ dived 
by the mean power between 0 and $125 \mathrm{~Hz}$. Cells were defined as 'theta modulated' if their TMI was greater than 5 .

\section{Analysis of speed modulated firing}

Speed modulation of single unit activity was calculated based on analysis described in Kropff et al., (2015). Running speed and firing rate of individual clusters were calculated for $40 \mathrm{~ms}$ bins of data and smoothed across 500 ms using a Gaussian window function. Running speeds from $2-30 \mathrm{~cm} / \mathrm{s}$ and containing more than $0.5 \%$ of total recording duration were used for further analysis. Speed modulation of cells was then defined by the correlation between all running speed and firing rate bins and a speed score calculated using the Fisher-z transformation of the correlation coefficient, r. Observed speed correlations were compared to a distribution of randomly sampled correlations of shuffled data. For shuffling, time stamps were forwardshifted by a pseudorandom period between $20 \mathrm{~s}$ and the total trial length minus $20 \mathrm{~s}$, with the end of the trial wrapped to the beginning and reanalysed using the method above. Cells were defined as 'speed modulated' if their speed score $(z)$ was greater than the $95^{\text {th }}$ percentile, or less than the $5^{\text {th }}$ percentile, of the global distribution of scores produced from at least 250 shuffled data sets for each unit (Figure 3).

\section{Analysis of head direction properties}

Head direction was determined by calculating the angle between two LEDs attached to the animal's headstage. Time periods where neither, or only one, of the LEDs were observed above threshold were discarded. Firing rate was calculated for $3^{\circ}$ bins of head direction and smoothed, using a Gaussian window over $14^{\circ}$. A 'head direction score' was defined as the resultant mean vector length, calculated from the smoothed firing rate maps. Observed mean vector length was also compared to the $95^{\text {th }}$ percentile of a distribution of shuffled data produced as above. 
Spike locations for each cell were obtained with a 2D histogram count, using the MATLAB

493 function histcounts2. Firing rate was calculated for $3 \times 3 \mathrm{~cm}$ bins across recording environments and smoothed using a 2D Gaussian function across 1.5 standard deviations. 'Gridness' was calculated using a 2D autocorrelation of smoothed firing rate maps ${ }^{5}$. Spatial periodicity was determined by rotating autocorrelations in steps of $30^{\circ}$, between the central peak and the 6 closest peaks, and correlating the rotated versions with the original. Grid score was expressed as the difference between rotations at $30^{\circ}, 90^{\circ}$ and $150^{\circ}$, where if firing maps show a hexagonal pattern give low correlations, and $60^{\circ}$ and $120^{\circ}$ where correlations will be high.

501 The spatial information content (SI) of each cell was defined using the measure described by

502 Skaggs et al., (1993) and expressed in terms of bits/spike. This approach measures the extent to which a cell's firing rate can be used to predict the animal's location. By definition, this does not assume spatial periodicity and has been used for quantifying place cell activity ${ }^{19,27,58,79}$ as well as spatially selective firing in the lateral $\mathrm{EC}^{80}$.

To control for changes in neuronal firing rate effecting coding properties, spike trains for each WT cell were uniformly downsampled incrementally between 1 and 100 times. Grid scores, mean vector lengths and speed scores were then calculated again for each case and compared to rTg4510 scores at equivalent firing rates.

514 Single unit and LFP data recorded from the CA1 pyramidal cell layer from previously published datasets were additionally analysed for speed encoding (Booth et al., (2016b)). These data 
were collected using microdrives containing independently adjustable tetrodes, while animals

517 ran on a linear track. Running speed - firing rate relationships were determined using the

518 processing pathway described above.

520 Electrode placement

521 At the end of experiments, mice received a lethal overdose of sodium pentobarbital (Euthetal)

522 and electrolytic lesions were made at several electrode locations across the recording array.

523 Mice were then transcardially perfused with $4 \% \mathrm{v} / \mathrm{v}$ formaldehyde in $0.1 \mathrm{M}$ phosphate buffered

524 saline (PBS). Brains were extracted from the skull and stored in $4 \%$ formaldehyde before being

525 cut in sagittal sections $(50 \mu \mathrm{m})$ using a vibratome (Leica VT1000) and stained with cresyl

526 violet. The position of electrode sites was determined from digital pictures taken with a $2.5 \mathrm{X}$

527 objective on a light microscope using QCapture pro 7 software (Qimaging). Probe electrode

528 location was expressed as distance from the most dorsal electrode site in layer II/III MEC. 


\section{Acknowledgments}

531 This work was supported by an Alzheimer's Research UK Major Project Grant awarded to

532 J.B. (ARUK-PG2017B-7). J.W. was an Alzheimer's Research UK Fellow (ARUK-RF2015-6).

533 A.R. was a Royal Society Industry Fellow. K.P. is an employee of Eli Lilly and Company. Eli

534 Lilly provided all the mice used in this study.

\section{Author Contributions}

536 Conceptualization, J.B., A.R., T.R; Methodology, K.P., T.R., J.W.; Investigation, T.R and

537 J.W.; Writing - Original Draft, T.R. and JB.; Writing -Review \& Editing, J.W., K.P., A.R., T.R.

538 and J.B.; Funding Acquisition, J.B., J.W., A.R.; Resources, J.B., A.R., K.P.; Supervision,

539 J.B., A.R.

540 Declaration of Interests

541 The authors declare no competing interests. 


\section{References}

1. O'Keefe, J. \& Dostrovsky, J. The hippocampus as a spatial map. Preliminary evidence from unit activity in the freely-moving rat. Brain Res. 34, 171-175 (1971).

2. Fyhn, M., Molden, S., Witter, M. P., Moser, E. I. \& Moser, M.-B. Spatial representation in the entorhinal cortex. Science 305, 1258-64 (2004).

3. Hafting, T., Fyhn, M., Molden, S., Moser, M.-B. B. \& Moser, E. I. Microstructure of a spatial map in the entorhinal cortex. Nature 436, 801-6 (2005).

4. Giocomo, L. M. et al. Topography of head direction cells in medial entorhinal cortex. Curr. Biol. 24, 252-62 (2014).

5. Sargolini, F. et al. Conjunctive representation of position, direction, and velocity in entorhinal cortex. Science 312, 758-62 (2006).

6. Ranck, J. . Head-direction cells in the deep cell layers of dorsal presubiculum in freely moving rats. in Society for Neuroscience Abstracts 10, (1984).

7. Valerio, S. \& Taube, J. S. Path integration: how the head direction signal maintains and corrects spatial orientation. Nat. Neurosci. 15, 1445-53 (2012).

8. Kropff, E., Carmichael, J. E., Moser, M.-B. \& Moser, E. I. Speed cells in the medial entorhinal cortex. Nature 523, 419-424 (2015).

9. Hinman, J. R., Brandon, M. P., Climer, J. R., Chapman, G. W. \& Hasselmo, M. E. Multiple Running Speed Signals in Medial Entorhinal Cortex. Neuron 91, 666-679 (2016).

562

10. Lever, C., Burton, S., Jeewajee, A., O’Keefe, J. \& Burgess, N. Boundary vector cells in the subiculum of the hippocampal formation. J. Neurosci. 29, 9771-7 (2009).

11. Solstad, T., Boccara, C. N., Kropff, E., Moser, M.-B. \& Moser, E. I. Representation of geometric borders in the entorhinal cortex. Science 322, 1865-8 (2008).

12. Barry, C. \& Bush, D. From A to Z: a potential role for grid cells in spatial navigation.

13. Allison, S. L., Fagan, A. M., Morris, J. C. \& Head, D. Spatial Navigation in Preclinical Neural Syst. Circuits 2, 6 (2012).

14. Lithfous, S., Dufour, A. \& Després, O. Spatial navigation in normal aging and the prodromal stage of Alzheimer's disease: Insights from imaging and behavioral studies. Ageing Res. Rev. 12, 201-213 (2013).

15. Hort, J. et al. Spatial navigation deficit in amnestic mild cognitive impairment. Proc.

16. Laczó, J. et al. Spatial navigation and APOE in amnestic mild cognitive impairment.

17. Mokrisova, I. et al. Real-space path integration is impaired in Alzheimer's disease and

18. Pooler, A. M. et al. Propagation of tau pathology in Alzheimer's disease: identification of novel therapeutic targets. Alzheimers. Res. Ther. 5, 49 (2013).

19. Booth, C. A. et al. Altered Intrinsic Pyramidal Neuron Properties and Pathway-Specific Synaptic Dysfunction Underlie Aberrant Hippocampal Network Function in a Mouse 
Model of Tauopathy. J. Neurosci. 36, 350-63 (2016).

20. Tamagnini, F. et al. Hippocampal neurophysiology is modified by a diseaseassociated C-terminal fragment of tau protein. Neurobiol. Aging 60, 44-56 (2017).

21. Brown, J. T., Chin, J., Leiser, S. C., Pangalos, M. N. \& Randall, A. D. Altered intrinsic neuronal excitability and reduced $\mathrm{Na}(+)$ currents in a mouse model of Alzheimer's disease. Neurobiol. Aging 32, 2109.e1-e2109.e14 (2011).

22. Kerrigan, T. L., Brown, J. T. \& Randall, A. D. Characterization of altered intrinsic excitability in hippocampal CA1 pyramidal cells of the A $\beta$-overproducing PDAPP mouse. Neuropharmacology 79, 515-524 (2014).

23. Hoover, B. R. et al. Tau Mislocalization to Dendritic Spines Mediates Synaptic Dysfunction Independently of Neurodegeneration. Neuron 68, 1067-1081 (2010).

24. Brown, J. T., Richardson, J. C., Collingridge, G. L., Randall, A. D. \& Davies, C. H. Synaptic transmission and synchronous activity is disrupted in hippocampal slices taken from aged TAS10 mice. Hippocampus 15, 110-117 (2005).

25. Fitzjohn, S. M. et al. Age-related impairment of synaptic transmission but normal longterm potentiation in transgenic mice that overexpress the human APP695SWE mutant form of amyloid precursor protein. J. Neurosci. 21, 4691-8 (2001).

26. Cheng, J. \& Ji, D. Rigid firing sequences undermine spatial memory codes in a neurodegenerative mouse model. Elife 2, e00647 (2013).

27. Cacucci, F., Yi, M., Wills, T. J., Chapman, P. \& O'Keefe, J. Place cell firing correlates with memory deficits and amyloid plaque burden in Tg2576 Alzheimer mouse model. Proc. Natl. Acad. Sci. 105, 7863-7868 (2008).

28. Booth, C. A. et al. Electrical and Network Neuronal Properties Are Preferentially Disrupted in Dorsal, But Not Ventral, Medial Entorhinal Cortex in a Mouse Model of Tauopathy. J. Neurosci. 36, 312-324 (2016).

29. Fu, H. et al. Tau Pathology Induces Excitatory Neuron Loss, Grid Cell Dysfunction, and Spatial Memory Deficits Reminiscent of Early Alzheimer's Disease. Neuron 93, 533-541.e5 (2017).

30. Kunz, L. et al. Reduced grid-cell-like representations in adults at genetic risk for Alzheimer's disease. Science (80-. ). 350, (2015).

31. McNaughton, B. L., Battaglia, F. P., Jensen, O., Moser, E. I. \& Moser, M.-B. B. Path integration and the neural basis of the 'cognitive map'. Nat. Rev. Neurosci. 7, 663-78 (2006).

32. Burak, Y., Fiete, I. R., Trappenberg, T., Araujo, I. de \& Wiggins, C. Accurate Path Integration in Continuous Attractor Network Models of Grid Cells. PLoS Comput. Biol. 5, e1000291 (2009).

33. Bush, D. \& Burgess, N. A hybrid oscillatory interference/continuous attractor network model of grid cell firing. J. Neurosci. 34, 5065-79 (2014).

34. Ahmed, O. J. \& Mehta, M. R. Running Speed Alters the Frequency of Hippocampal Gamma Oscillations. J. Neurosci. 32, (2012).

35. McFarland, W. L., Teitelbaum, H. \& Hedges, E. K. Relationship between hippocampal theta activity and running speed in the rat. J. Comp. Physiol. Psychol. 88, 324-8 (1975).

36. Zheng, C., Bieri, K. W., Trettel, S. G. \& Colgin, L. L. The relationship between gamma 
frequency and running speed differs for slow and fast gamma rhythms in freely behaving rats. Hippocampus 25, 924-38 (2015).

37. Chen, Z., Resnik, E., McFarland, J. M., Sakmann, B. \& Mehta, M. R. Speed controls the amplitude and timing of the hippocampal gamma rhythm. PLoS One 6, e21408 (2011).

38. Sławińska, U. \& Kasicki, S. The frequency of rat's hippocampal theta rhythm is related to the speed of locomotion. Brain Res. 796, 327-331 (1998).

39. Ramsden, M. et al. Age-dependent neurofibrillary tangle formation, neuron loss, and memory impairment in a mouse model of human tauopathy (P301L). J. Neurosci. 25, 10637-47 (2005).

40. Selenica, M.-L. et al. Histone deacetylase 6 inhibition improves memory and reduces total tau levels in a mouse model of tau deposition. Alzheimers. Res. Ther. 6, 12 (2014).

41. Cook, C. et al. Severe amygdala dysfunction in a MAPT transgenic mouse model of frontotemporal dementia. Neurobiol. Aging 35, 1769-77 (2014).

42. Jul, P. et al. Hyperactivity with Agitative-Like Behavior in a Mouse Tauopathy Model. J. Alzheimer's Dis. 49, 783-795 (2015).

43. Langston, R. F. et al. Development of the Spatial Representation System in the Rat. Science (80-. ). 328, (2010).

44. Buetfering, C., Allen, K. \& Monyer, H. Parvalbumin interneurons provide grid celldriven recurrent inhibition in the medial entorhinal cortex. Nat. Neurosci. 17, 710-8 (2014).

45. Skaggs, W. E., Mcnaughton, B. L., Gothard, K. M. \& Markus, E. J. An InformationTheoretic Approach to Deciphering the Hippocampal Code. Neural Inf. Process. Syst. 1030-1037 (1993).

46. McNaughton, B. L., Barnes, C. A. \& O’Keefe, J. The contributions of position, direction, and velocity to single unit activity in the hippocampus of freely-moving rats. Exp. Brain Res. 52, 41-49 (1983).

47. Lu, X. \& Bilkey, D. K. The velocity-related firing property of hippocampal place cells is dependent on self-movement. Hippocampus 20, NA-NA (2009).

48. Bonnevie, T. et al. Grid cells require excitatory drive from the hippocampus. Nat. Neurosci. 16, 309-317 (2013).

49. Burgess, N., Barry, C. \& O’Keefe, J. An oscillatory interference model of grid cell firing. Hippocampus 17, 801-12 (2007).

50. Brandon, M. P. et al. Reduction of theta rhythm dissociates grid cell spatial periodicity from directional tuning. Science 332, 595-9 (2011).

51. Koenig, J., Linder, A. N., Leutgeb, J. K. \& Leutgeb, S. The Spatial Periodicity of Grid Cells Is Not Sustained During Reduced Theta Oscillations. Science (80-. ). 332, (2011).

52. Winter, S. S., Clark, B. J. \& Taube, J. S. Disruption of the head direction cell network impairs the parahippocampal grid cell signal. Science (80-. ). 347, (2015).

53. Santacruz, K. et al. Tau suppression in a neurodegenerative mouse model improves memory function. Science 309, 476-81 (2005). 
54. Spires, T. L. et al. Region-specific dissociation of neuronal loss and neurofibrillary pathology in a mouse model of tauopathy. Am. J. Pathol. 168, 1598-607 (2006).

55. Blackmore, T. et al. Tracking progressive pathological and functional decline in the rTg4510 mouse model of tauopathy. Alzheimers. Res. Ther. 9, 77 (2017).

56. Hales, J. B. et al. Medial Entorhinal Cortex Lesions Only Partially Disrupt Hippocampal Place Cells and Hippocampus-Dependent Place Memory. Cell Rep. 9, 893-901 (2014).

57. Van Cauter, T., Poucet, B. \& Save, E. Unstable CA1 place cell representation in rats with entorhinal cortex lesions. Eur. J. Neurosci. 27, 1933-46 (2008).

58. Brun, V. H. et al. Impaired Spatial Representation in CA1 after Lesion of Direct Input from Entorhinal Cortex. Neuron 57, 290-302 (2008).

59. Wills, T. J., Barry, C. \& Cacucci, F. The abrupt development of adult-like grid cell firing in the medial entorhinal cortex. Front. Neural Circuits 6, 21 (2012).

60. Muessig, L., Hauser, J., Wills, T. J. \& Cacucci, F. A Developmental Switch in Place Cell Accuracy Coincides with Grid Cell Maturation. Neuron 86, 1167-73 (2015).

61. Fuhs, M. C. \& Touretzky, D. S. A Spin Glass Model of Path Integration in Rat Medial Entorhinal Cortex. J. Neurosci. 26, (2006).

62. Etienne, A. S. \& Jeffery, K. J. Path integration in mammals. Hippocampus 14, 180 192 (2004).

63. Allen, K. et al. Impaired Path Integration and Grid Cell Spatial Periodicity in Mice Lacking GluA1-Containing AMPA Receptors. J. Neurosci. 34, (2014).

64. Justus, D. et al. Glutamatergic synaptic integration of locomotion speed via septoentorhinal projections. Nat. Neurosci. 20, 16-19 (2016).

65. Taube, J. S. The Head Direction Signal: Origins and Sensory-Motor Integration. Annu. Rev. Neurosci. 30, 181-207 (2007).

66. Stackman, R. W. \& Taube, J. S. Firing properties of rat lateral mammillary single units: head direction, head pitch, and angular head velocity. J. Neurosci. 18, 9020-37 (1998).

67. Blair, H. T., Welday, A. C. \& Zhang, K. Scale-invariant memory representations emerge from moiré interference between grid fields that produce theta oscillations: a computational model. J. Neurosci. 27, 3211-29 (2007).

68. Sharp, P. E., Tinkelman, A. \& Cho, J. Angular velocity and head direction signals recorded from the dorsal tegmental nucleus of gudden in the rat: Implications for path integration in the head direction cell circuit. Behav. Neurosci. 115, 571-588 (2001).

69. Colgin, L. L. et al. Frequency of gamma oscillations routes flow of information in the hippocampus. Nature 462, 353-7 (2009).

70. Chrobak, J. J. \& Buzsaki, G. Gamma Oscillations in the Entorhinal Cortex of the Freely Behaving Rat. J. Neurosci. 18, 388-398 (1998).

71. Kemere, C., Carr, M. F., Karlsson, M. P. \& Frank, L. M. Rapid and continuous modulation of hippocampal network state during exploration of new places. PLoS One 8, e73114 (2013).

72. Jul, P. et al. Hyperactivity with Agitative-Like Behavior in a Mouse Tauopathy Model. J. Alzheimer's Dis. 49, 783-795 (2015). 
73. Cheng, D., Low, J. K., Logge, W., Garner, B. \& Karl, T. Novel behavioural characteristics of female APPSwe/PS1 $1 \mathrm{E} 9$ double transgenic mice. Behav. Brain Res. 260, 111-118 (2014).

74. Przybyla, M. et al. Disinhibition-like behavior in a P301S mutant tau transgenic mouse model of frontotemporal dementia. Neurosci. Lett. 631, 24-29 (2016).

75. Rossant, C. et al. Spike sorting for large, dense electrode arrays. Nat. Neurosci. 19, 634-41 (2016).

76. Beed, P. et al. Inhibitory gradient along the dorsoventral axis in the medial entorhinal cortex. Neuron 79, 1197-207 (2013).

77. Miettinen, M., Koivisto, E., Riekkinen, P. \& Miettinen, R. Coexistence of parvalbumin and GABA in nonpyramidal neurons of the rat entorhinal cortex. Brain Res. 706, 113122 (1996).

78. Wills, T. J., Cacucci, F., Burgess, N. \& O'Keefe, J. Development of the hippocampal cognitive map in preweanling rats. Science 328, 1573-6 (2010).

79. Hussaini, S. A., Kempadoo, K. A., Thuault, S. J., Siegelbaum, S. A. \& Kandel, E. R. Increased size and stability of CA1 and CA3 place fields in HCN1 knockout mice. Neuron 72, 643-53 (2011).

80. Deshmukh, S. S. \& Knierim, J. J. Representation of non-spatial and spatial information in the lateral entorhinal cortex. Front. Behav. Neurosci. 5, 69 (2011). 
A

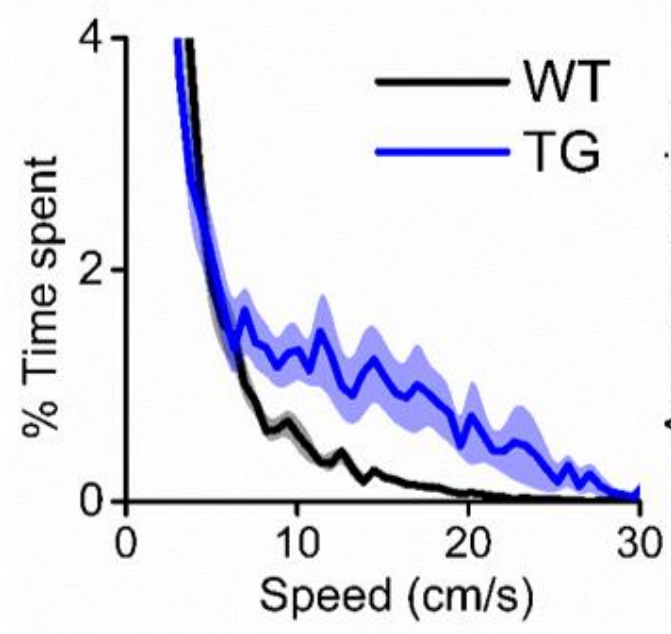

B

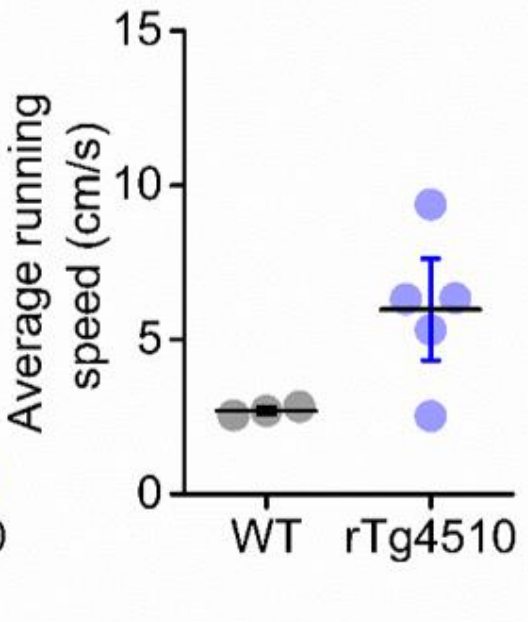

Figure S1: rTg4510 mice display hyperactive phenotype. A) Breakdown of animals time spent at eat running speed, showing rTg4510 mice spend more time moving at higher velocities. B) Average running speed across (mean $\pm \mathrm{SEM}$ ) entire recording session is greater in $r$ Tg4510 mice ( $P=0.04$, Unpaired T-test). 
A
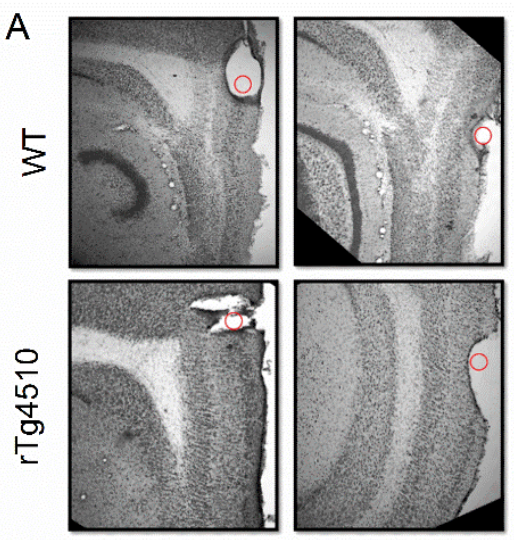

$\mathrm{Bi}$
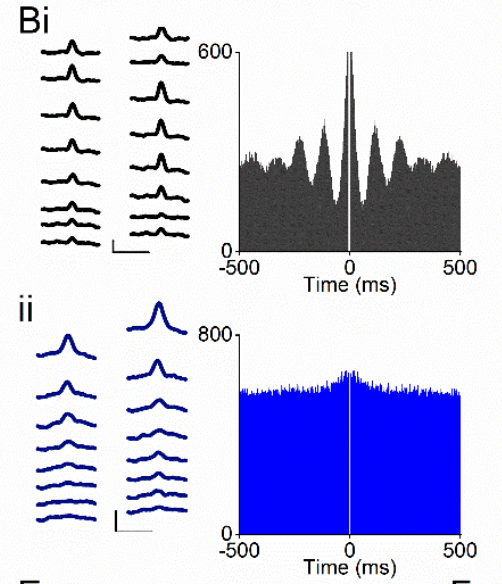

$\mathrm{E}$

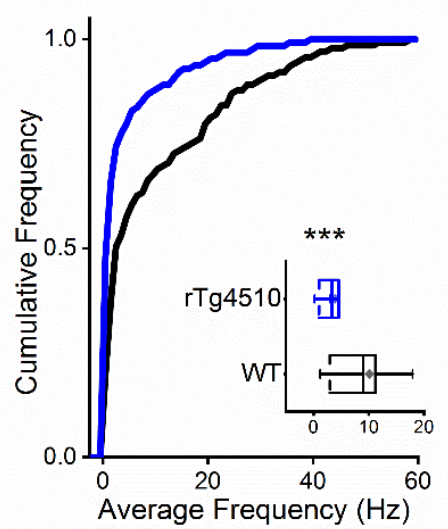

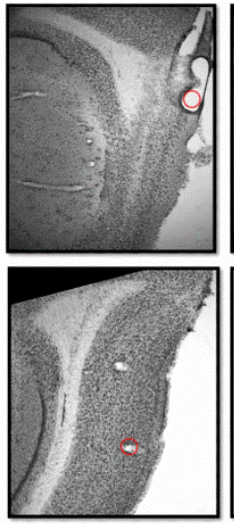
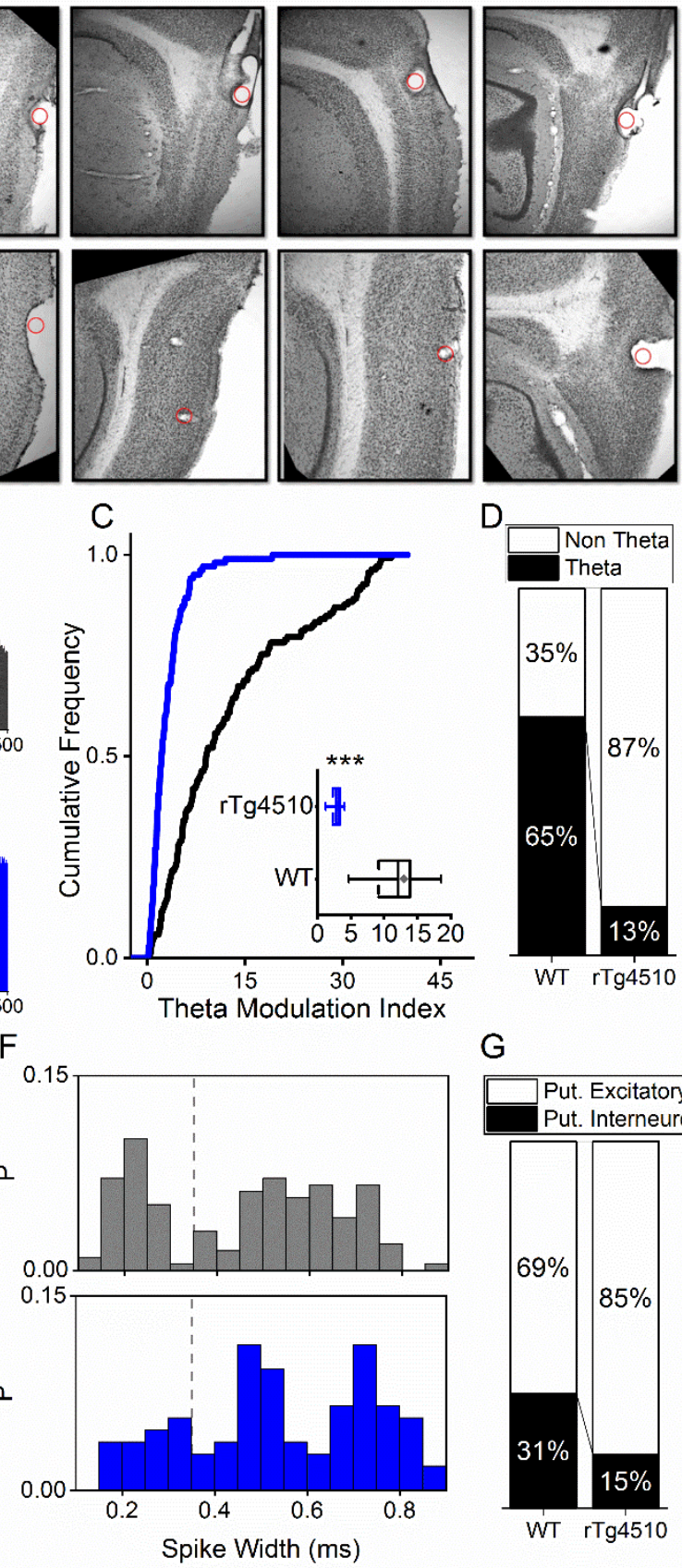

G

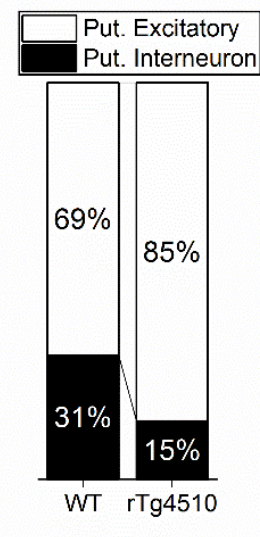

Figure S2: Firing properties of mEC single units. A) Example sections showing electrode lesions (red dot) of final probe location in mEC of WT and $\mathrm{rTg} 4510$ mice. B) Average waveforms from an example cell recorded from a 16 channel silicone probe shank for WT (i) and $r \operatorname{Tg} 4510$ (ii) mouse, with firing autocorrelations. Scale bars: $0.4 \mathrm{~ms}, 50 \mu \mathrm{V}$. C) Cumulative frequency plot of theta modulation index for all recorded mEC single units, with average modulation for WT (black) and rTg4510 (blue) mice inset. D) Proportion of cells displaying theta modulation (threshold: TMl>5). E) Average firing frequency across entire recording session of mEC neurons for WT (black) and rTg4510 (blue) mice, average inset. F) Spikewidth probability histogram for WT (black) and rTg4510 (blue) units. G) Proportion of cells classified as putative interneurons (spike-width $<0.35 \mathrm{~ms}$, dotted line in E) and putative excitatory. Box plots: dotted line: median, diamond: mean \pm SEM, whiskers: $25^{\text {th }} / 75^{\text {th }}$ centile), ${ }^{* * *} \mathrm{p}<0.001$ Mann-Whitney U test. 
$\mathrm{Ai}$

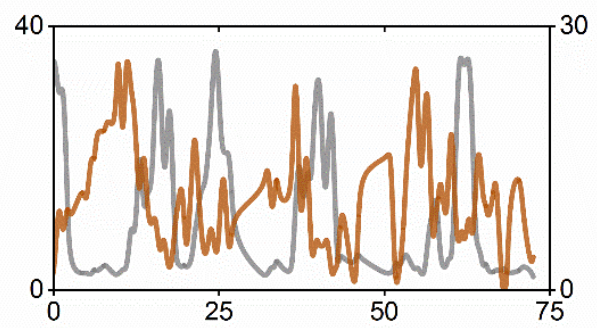

ii

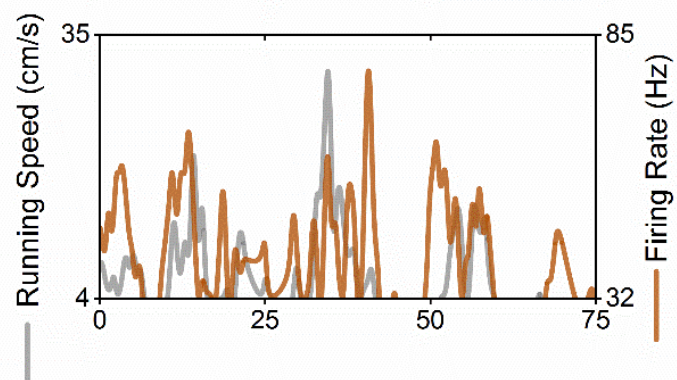

iii

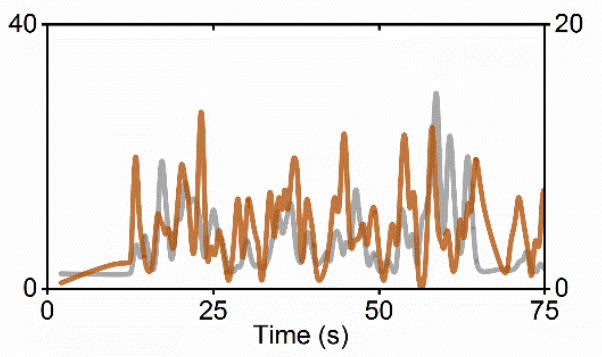

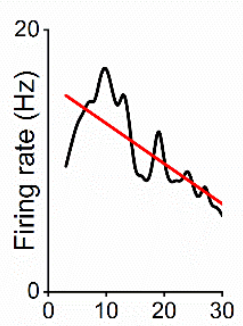
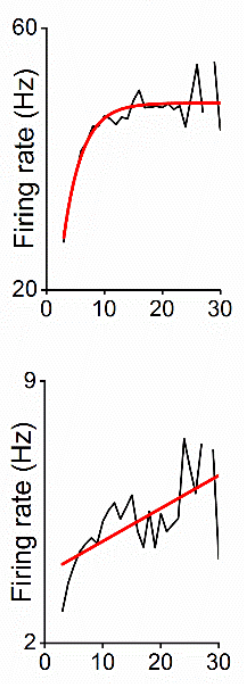

Runnina speed $(\mathrm{cm} / \mathrm{s})$
B

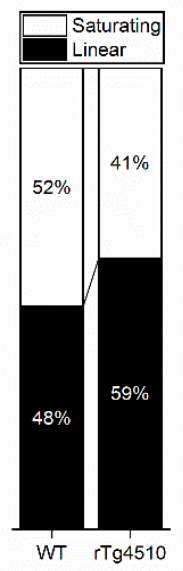

C

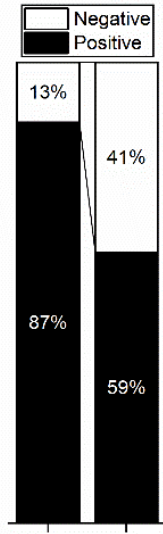

iv WT rTg $^{2} 510$

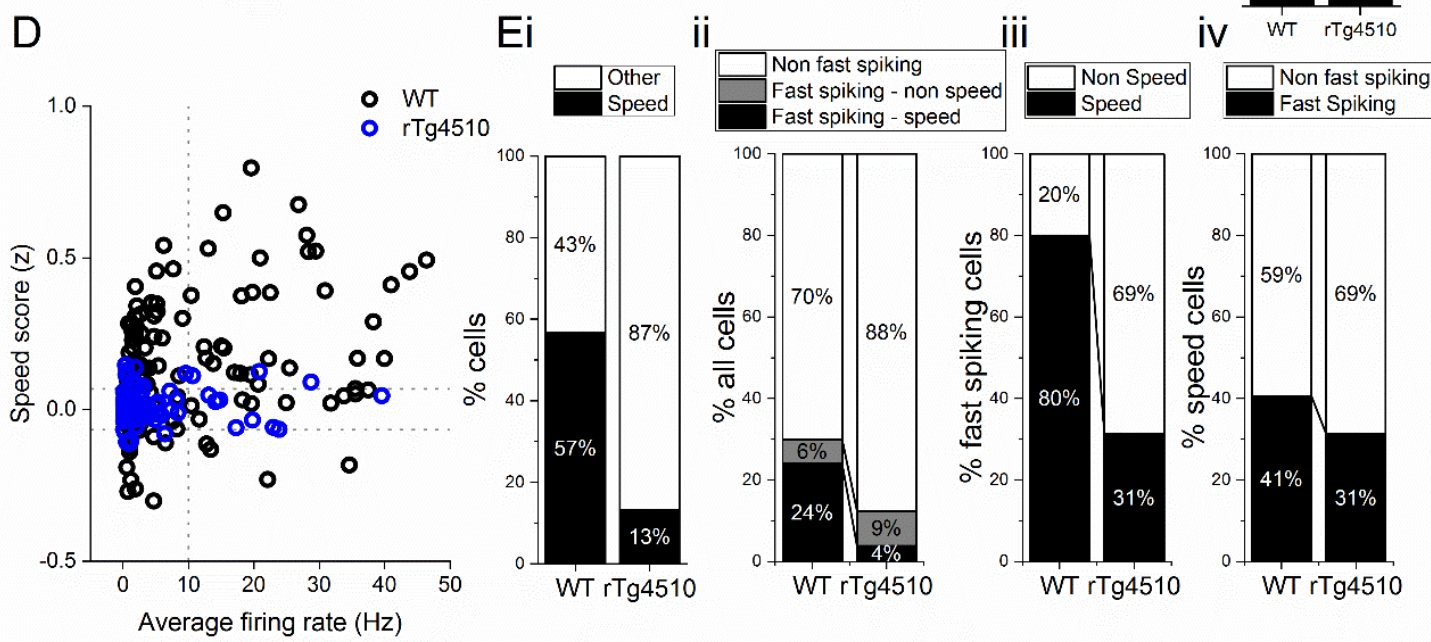

Figure S3: Properties of MEC speed modulated cells. A) Example negative (i), saturating (ii) and linear (iii) speed-modulated cells, with running speed (grey) and cell firing rate (orange), with average for each speed bin $(1 \mathrm{~cm} / \mathrm{s})$ (right). Red line: linear/exponential fit for each. B) Proportion of speed modulated mEC cells that are best described by linear and saturating fits for WT and rTg4510 mice. C) Proportion of speed modulated units that show positive ( $>95^{\text {th }}$ centile of shuffled distribution) and negative $\left(<5^{\text {th }}\right.$ centile of shuffled distribution) speed modulation. D) Plot of speed score vs average firing rate, showing faster-spiking cells are more likely to have high speed modulation in WT, but not rTg4510 mice. Ei) Proportion of speed-modulated cells in WT and rTg4510 mice (replotted from Fig 3E). ii) Proportions of cells classified as fast-spiking $(>10 \mathrm{~Hz})$, with breakdown into speed and non-speed modulators for each genotype. iii) Proportion of fast spiking cells which display speed modulation, which shows decreased numbers in rTg4510 mice compared to WT. iv) Proportion of speed modulated cells which are classified as fast spiking shows only small decrease in $\mathrm{rTg} 4510$ mice. 

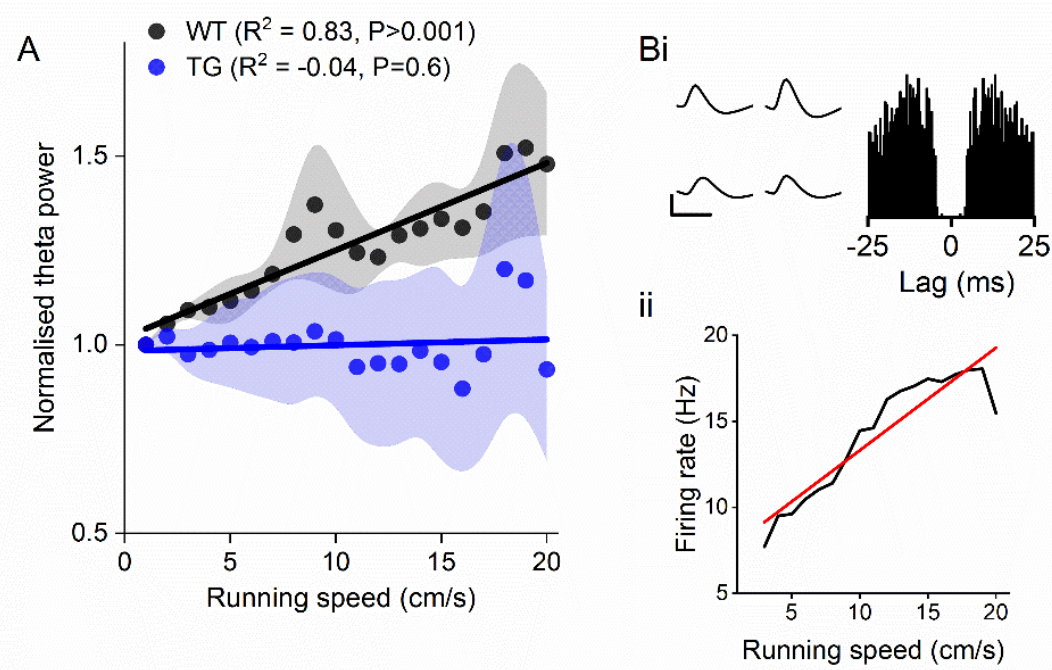

iii
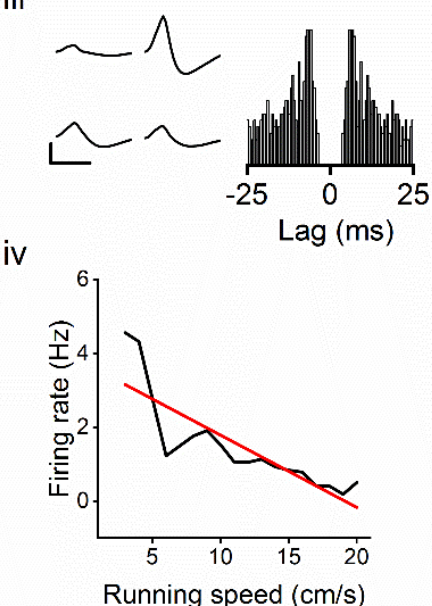

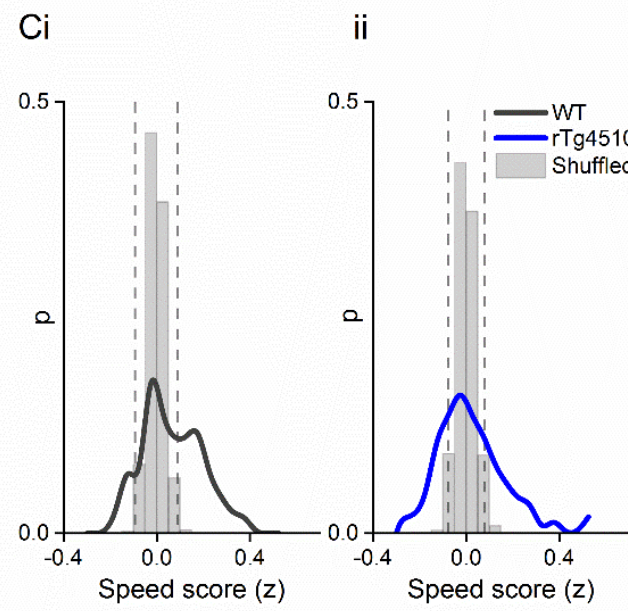

D
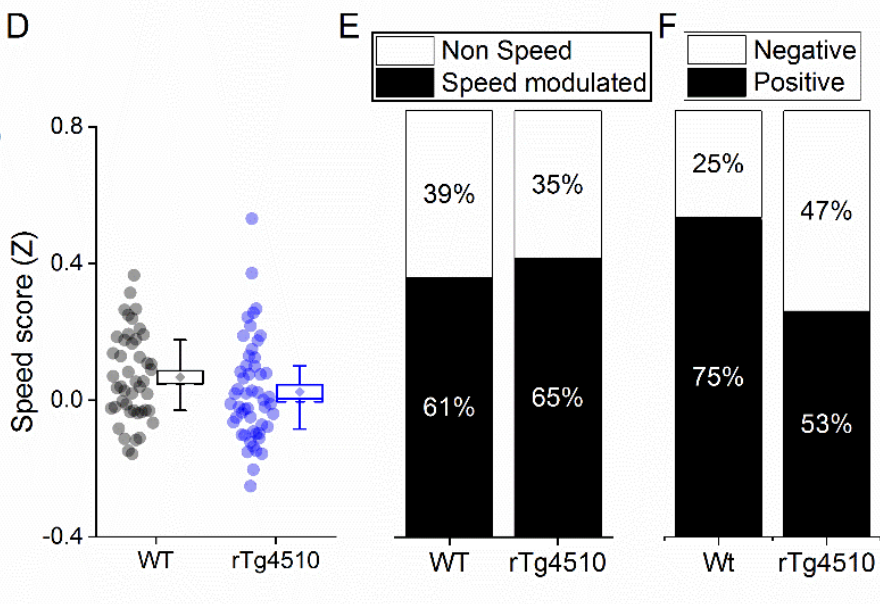

Figure S4: Speed modulation of single units in hippocampal CA1 pyramidal cell layer also shows similar increase in negatively speed modulated firing. A) Normalised theta oscillation amplitude from WT and $\mathrm{rTg} 4510$ mice with increasing running speed, with average linear regression above $\left(W T: R^{2}=0.83, p<0.001, n=6\right.$ mice; $r T g 4510: R^{2}=-0.04, p=0.6, n$ $=4$ mice). B) Example speed-modulated cells recorded from the CA1 pyramidal cell layer. The cell on the left (i and ii) was positively speed-modulated, whilst the cell on the right (iii and iv) was negatively speed-modulated. C) histograms of speed scores for WT and rTg4510 mice. $5^{\text {th }}$ and $95^{\text {th }}$ centiles of shuffled data shown as dotted lines. D) box plot comparing speed scores between the two genotypes E) Proportion of cells passed criteria for speed modulated firing is approximately even between genotypes (WT: 25/46 units, rTg4510: 27/52 units). F) Increased proportion of negatively speed modulated units in CA1 in rTg4510 mice, compared to WT controls (WT: 5/25 units, rTg4510:13/27 units; $X^{2}(1)=4.5, p=0.03$, Chi-Square test). 
$\mathrm{Ai}$

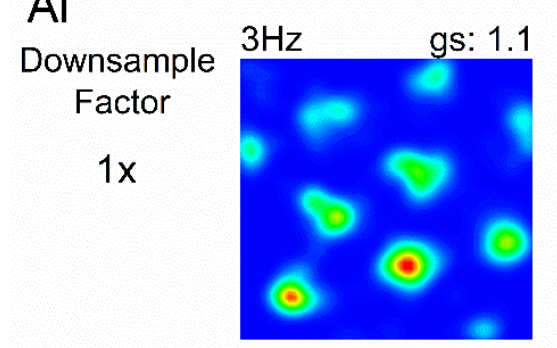

$10 x$
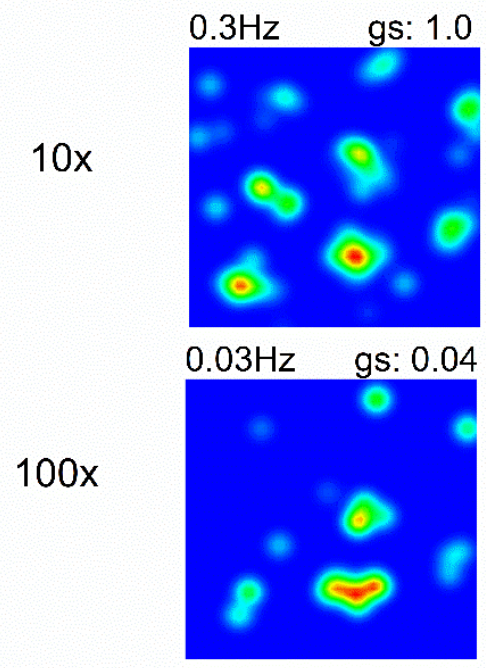

B

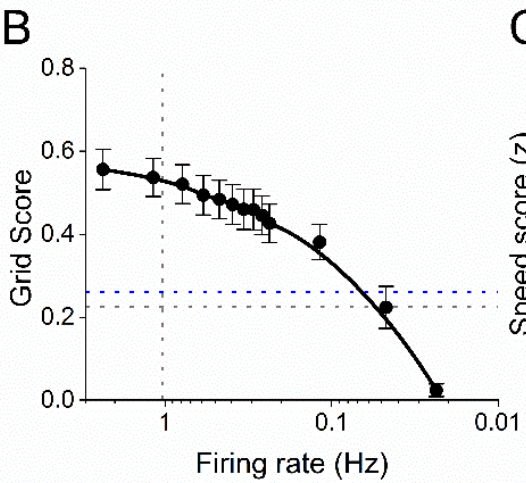

ii
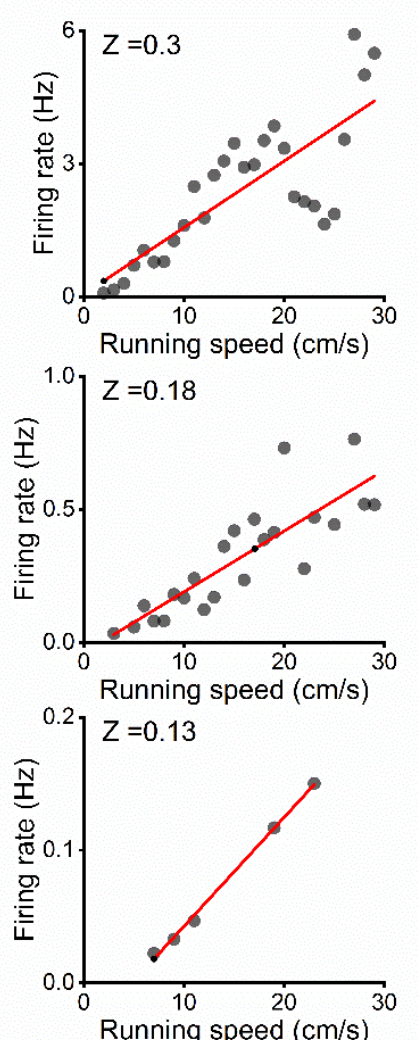

C

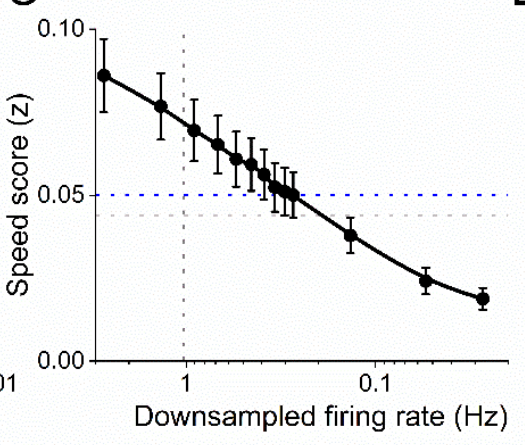

iii
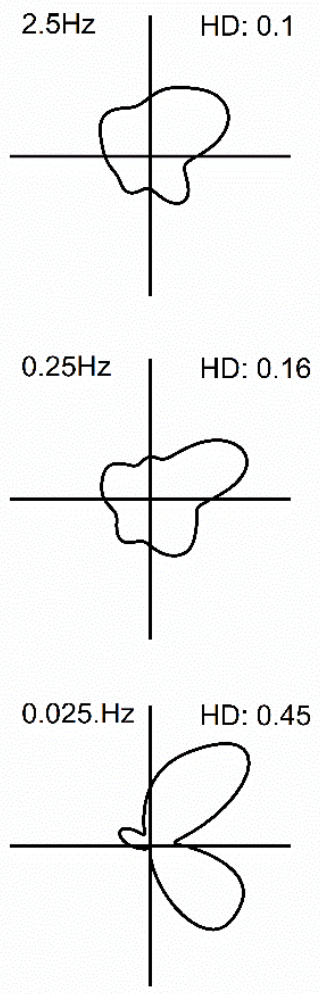

Figure S5: Decreased firing rate does not account for changes in spatial metrics. A) Example grid (i), speed (ii) and head-direction (iii) cell firing rate maps, with progressive down sampling of spikes. B) Effect of down sampled spike trains on grid score output on WT neurons. Horizontal line: grid score threshold from $95^{\text {th }}$ centile of shuffled distributions for WT (grey) and rTg4510 (blue) mice. Vertical line: Average firing of rTg4510 MEC neurons. C) Effect of down sampled spike trains on speed score output on WT neurons. Horizontal line: grid score threshold from $95^{\text {th }}$ centile of shuffled distributions. Vertical line: Average firing of rTg4510 MEC neurons. D) Effect of down sampled spike trains on head-direction score output on WT neurons. Horizontal line: grid score threshold from $95^{\text {th }}$ centile of shuffled distributions. Vertical line: Average firing of rTg4510 MEC neurons. 


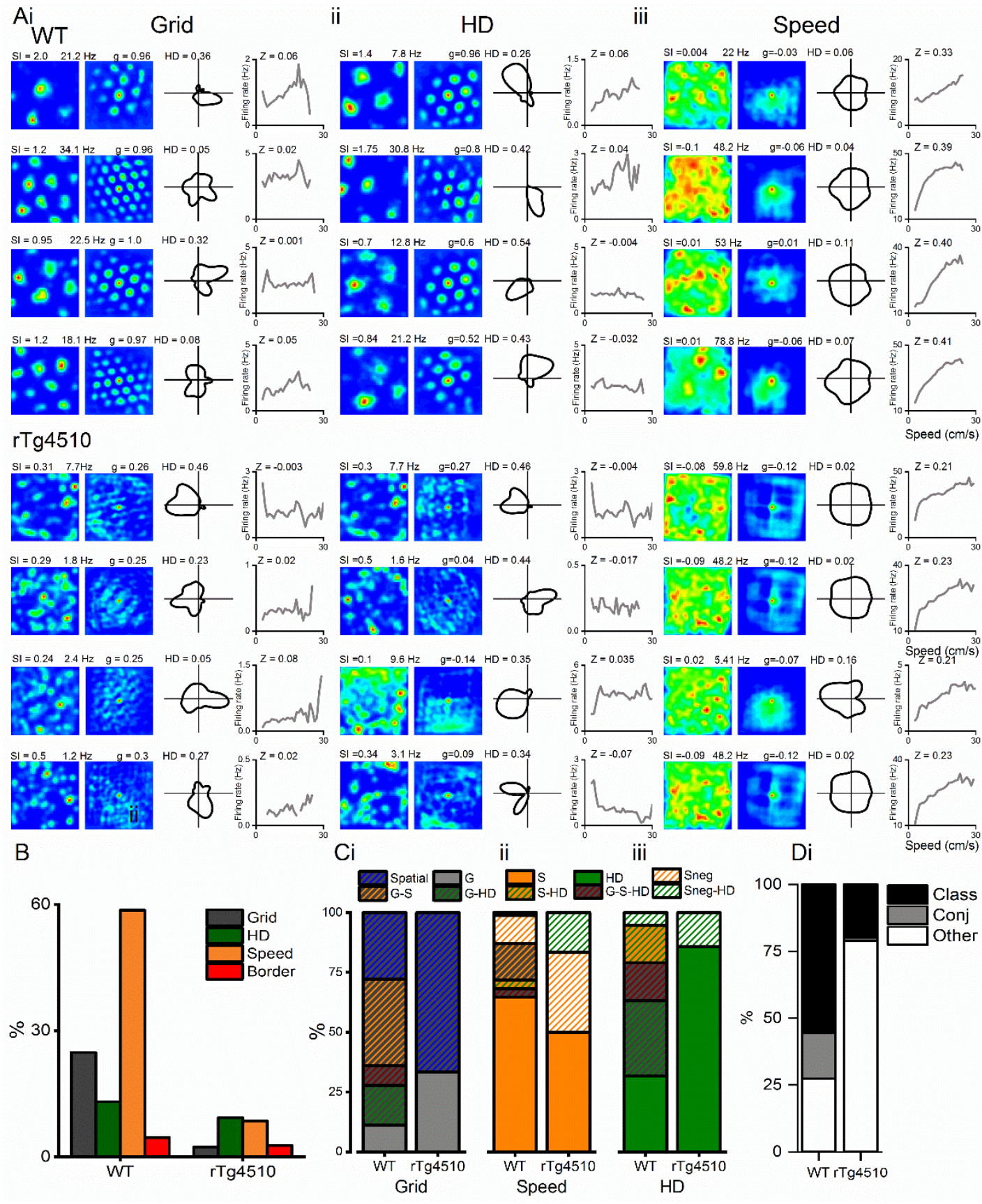

Figure S6: Conjunctive representation of grid, head direction and running speed in WT and rTg4510 mice. A) Example grid (i), head direction (ii) and speed (iii) cells taken from the highest modulation scores from WT (top) and rTg4510 (bottom) mice, showing spatial firing patterns with 2D spatial autocorrelation, head direction tuning and running speed-firing rate relationship and corresponding score (SI: spatial information, HD: mean vector length, Z: speed score). B) Proportions of grid, head-direction and speed modulated cells recorded in $\mathrm{mEC}$, showing reduced number of grid and speed, but not head-direction, cells passing threshold (95\% centile of shuffled distribution). C) Conjunctive proportions of grid cells (i), head direction cells (ii) and speed modulated cells (iii) recorded from WT and rTg4510 mice scaled to $100 \%$. D) Breakdown of units from WT and rTg4510 mice that satisfied a single criteria (class), multiple criteria (conj) or no discernible firing pattern (other). Key, G: grid, S: speed, HD: head direction, G-S: grid-speed, G-HD: grid-head direction, G-S-HD: grid-speedhead direction, SnegHD: negative speed-head-direction, Sneg: negative speed, Class: classed as cell type, Conj: conjunctive representation (more than 1 classification), other: not classified as grid, speed or HD. 